\title{
MIRROR SYMMETRY FOR LATTICE POLARIZED K3 SURFACES
}

\author{
Igor V. Dolgachev * \\ Department of Mathematics, University of Michigan, Ann Arbor, MI 48109
}

Introduction. There has been a recent explosion in the number of mathematical publications due to the discovery of a certain duality between some families of Calabi-Yau threefolds made by a group of theoretical physicists (see $[\mathbf{1 1 , 2 6 ]}$ for references). Roughly speaking this duality, called mirror symmetry, pairs two families $\mathcal{F}$ and $\mathcal{F}^{*}$ of Calabi-Yau threefolds in such a way that the following properties are satisfied:

MS1 The choice of the mirror family $\mathcal{F}^{*}$ involves the choice of a boundary point $\infty$ of a compactification $\overline{\mathcal{F}}$ of the moduli space for $\mathcal{F}$ at which the monodromy is "maximally unipotent".

MS2 For each $V \in \mathcal{F}$ and $V^{\prime} \in \mathcal{F}^{*}$ the Hodge numbers satisfy

$$
h^{1,1}(V)=h^{2,1}\left(V^{\prime}\right), \quad h^{2,1}(V)=h^{1,1}\left(V^{\prime}\right) .
$$

MS3 For some open subset $U$ of $\infty$, for any $V \in U \cap \mathcal{F}$, the Laurent expansion of the canonical symmetric trilinear form $S^{3}\left(H^{1}\left(V, \Theta_{V}\right)\right) \rightarrow H^{0,3}(V)^{\otimes 2}$ (the Griffiths-Yukawa cubic) at $\infty$ can be identified, after some special choice of local parameters and a basis of $H^{0,3}(V)$, with the quantum intersection form on $H^{2}\left(V^{\prime}\right), V^{\prime} \in \mathcal{F}^{*}$.

MS4 The period map induces a holomorphic multivalued mapping from the subset $U \cap \mathcal{F}$ to the tube domain $H^{2}\left(V^{\prime}, \mathbf{R}\right)+i \mathcal{K}_{V^{\prime}}$ where $\mathcal{K}_{V^{\prime}}$ is the Kähler cone of $V^{\prime} \in \mathcal{F}^{*}$ (the mirror mapping).

Although known to some experts but never stated explicitly, it is a fact that mirror symmetry is a very beautiful and non-trivial (in many respects still hypothetical) generalization to the next dimension of the duality for K3 surfaces discovered almost 20 years ago by H. Pinkham [34] and independently by the author and V. Nikulin $[\mathbf{8 , 9 , 3 1 ]}$. This duality was used to explain Arnold's Strange Duality for exceptional unimodal critical points [1]. There are repeated hints on the relationship between the latter duality and the mirror symmetry both in physics literature $([\mathbf{2 , 1 4 , 2 3 ]})$ and in mathematics literature $([\mathbf{6}, \mathbf{1 9}, \mathbf{3 5}, \mathbf{4 1}])$. Some of the results of this paper were independently obtained in $[\mathbf{3 , 1 9 , 2 1 , 2 7 , 3 5 ]}$ and some must be known to V. Batyrev and V. Nikulin. The paper [40]

* Research supported in part by a NSF grant. 
of Todorov is probably most relevant. Nevertheless I believe that it is worthwhile to give a detailed account of how the ideas of Arnold's Strange Duality allows one to state (and prove) precise analogs of properties MS1-MS4 for K3 surfaces.

Note that property MS2 says that the local moduli number of $V \in \mathcal{F}$ is equal to the second Betti number of $V^{\prime} \in \mathcal{F}^{*}$. In the case of K3 surfaces, the first number is always equal to 20 , and the second number is equal to 22 . The key observation is that in three-dimensional case the second Betti number is equal to the rank of the Picard group of algebraic cycles. This suggests to create different moduli families of K3 surfaces with condition on the Picard group. The simplest realization of this idea is based on the notion of a polarized K3 surface. This is pair $(X, h)$ where $X$ is a $\mathrm{K} 3$ surface and $h \in \operatorname{Pic}(X)$ is an ample (or pseudo-ample) divisor class. A generalization of this notion, due to $\mathrm{V}$. Nikulin [30], is the notion of a lattice polarized K3 surface. We fix a lattice $M$ (a free abelian group equipped with an integral quadratic form) and consider a pair $(X, j)$ where $X$ is a K3 surface, and $j: M \rightarrow \operatorname{Pic}(X)$ is a primitive embedding of lattices such that $j(M)$ contains a pseudo-ample divisor class. One can construct a coarse moduli space $\mathbf{K}_{M}$ of $M$-polarized K3 surfaces. An obvious condition of its non-emptiness is that $M$ is isomorpic to a sublattice of an even unimodular lattice $L$ of signature $(3,19)$ isomorphic to the second cohomology group of a K3 surface equipped with the cup-product.

To define the mirror family, we choose an isotropic primitive vector $f$ in the orthogonal complement $M^{\perp}$ of $M$ in $L$, and consider the lattice $\check{M}=(\mathbf{Z} f)_{M^{\perp}}^{\perp} / \mathbf{Z} f$. Under certain arithmetic conditions on $M$, the lattice $\check{M}$ admits a primitive embedding in $M^{\perp}$, and we can define the mirror moduli space $\mathbf{K}_{\check{M}}$. Additional conditions on $M$ ensure that the moduli spaces $\mathbf{K}_{M}$ and $\mathbf{K}_{\check{M}}$ are defined uniquely up to isomorphism, and $\check{\check{M}}=M$. Now if we have any complete family $\mathcal{F}$ of pseudo-ample $M$-polarized K3 surfaces its mirror family is any complete family of pseudo-ample $\check{M}$-polarized K3 surfaces.

It turns out that the choice of the isotropic vector $f$ is the analog of MS1. Property MS2 becomes

MS2' The dimension of the family $\mathcal{F}$ is equal to the rank of the Picard group of a general member from the mirror family $\mathcal{F}^{*}$.

In the case of K3 surfaces the Griffiths-Yukawa cubic becomes a quadratic map

$$
S^{2}\left(H^{1}\left(V, \Theta_{V}\right)\right) \rightarrow H^{0,2}(V)^{\otimes 2}
$$

and we have the following analog of MS3:

MS3' For some open subset $U$ of $\mathcal{F}$ near the boundary point (determined by the choice of isotropic vector $f$ ), for any $V \in U$, the Griffiths-Yukawa quadratic map can be canonically identified, after some choice of basis of $H^{0,2}(V)$, with the quadratic form on $\check{M} \otimes \mathbf{C}$.

Note that in our case the Griffiths-Yukawa quadratic map becomes the "constant" quantum intersection form and does not carry any information about rational curves on the mirror surfaces. This agrees with the fact that the quantum cohomology ring of a K3 surface coincides with the usual cohomology ring [36].

The next property is a very close analog of MS4:

MS4' The period map induces a holomorphic multivalued mapping from the subset $U$ from $\mathrm{MS3}^{\prime}$ to the tube domain $\operatorname{Pic}\left(V^{\prime}\right)_{\mathbf{R}}+\sqrt{-1} C(X)^{+} \subset \operatorname{Pic}\left(V^{\prime}\right)_{\mathbf{C}}$ where $C(X)^{+}$is the ample cone of $V^{\prime} \in \mathcal{F}^{*}$. It is called the mirror map. 
We also produce some computational evidence to support our point. The mirror candidates for a family of Calabi-Yau three-dimensional hypersurfaces in toric Fano varieties are obtained by Batyrev's construction from [5]. When this construction applies to K3 surfaces it is "often", but not always, gives our mirror family. This was first noticed by Batyrev in a preprint version of [5]. For example, in the case when $\mathcal{F}$ is the moduli family of quartic surfaces $(M=\mathbf{Z} e,(e, e)=4)$ the mirror family $\mathcal{F}^{*}$ is the one-dimensional family of K3 surfaces obtained by dividing the surfaces

$$
\lambda x_{0} x_{1} x_{2} x_{3}+x_{0}^{4}+x_{1}^{4}+x_{2}^{4}+x_{3}^{4}=0
$$

by a symplectic action of the group $(\mathbf{Z} / 4)^{2}$. Note the analogy with Green-Plesser's first discovered example of mirror symmetry for quintic hypersurfaces (see [11], pp.1-30). In this case assertion MS3' was verified in [27].

Other examples of our computations include the mirror families for the families of polarized K3 surfaces of degree $2 n$, for K3-covers of Enriques surfaces and Kummer surfaces, and the fourteen families coming from exceptional unimodal surface singularites. In the case of polarized K3 surfaces of degree $n$ we compute the monodromy group of the period and mirror mappings. By other methods this computation was done in [27] $(n=4)$ and [21] $(n=2,4,6)$. We prove that the mirror moduli space is isomorphic to the modular curve $X_{0}(n)^{+}=H / \Gamma_{0}(n)^{+}$, where $\Gamma_{0}(n)^{+}$is the Fricke double extension of the modular group $\Gamma_{0}(n)$. We relate the surfaces from this family to the $\operatorname{Kummer}$ surfaces $\operatorname{Kum}\left(E \times E^{\prime}\right)$, where $\left(E, E^{\prime}\right)$ is a pair of isogeneous elliptic curve.

There is another view of mirror symmetry more relevant to the string theory. Here one considers the moduli space of pairs $(V, \alpha)$ where $V$ is a Calabi-Yau manifold, and $\alpha=B+i K \in H^{2}(V, \mathbf{R} / \mathbf{Z})+i \mathcal{K}_{V}$ with $K$ equal to a Kähler form on $V$ with respect to the complex structure of $V$. Then the mirror mapping extends to a map of this space to itself defined on pairs $(V, \alpha)$ such that $V$ is close to a boundary point of the moduli space of complex structures and the imaginary part of $\alpha$ can be represented by the Einstein-Kähler metric of sufficiently large volume. The work of P. Aspinwal and D. Morrison [2,3] treats mirror symmetry for K3 surfaces from this point of view.

My acknowledgments go to V. Batyrev, D. Morrison, V. Nikulin and A. Todorov who shared my belief that Arnold's Strange Duality must be the pre-history of mirror symmetry and left it up to me to elaborate on this subject. I am also grateful to V. Batyrev, A. Greenspoon and D. Morrison for providing some references to the relevant literature.

1. Lattice polarized K3 surfaces. Let $X$ be a complex algebraic K3 surface, a nonsingular projective algebraic surface over $\mathbf{C}$ with vanishing canonical class and first Betti number. Via interesection form the second cohomology group $H_{2}(X, \mathbf{Z})$ is equipped with the structure of a lattice ( = a free abelian group together with an integral symmetric bilinear form on it). It is isomorphic to the lattice $L$ equal to the orthogonal sum of three copies of the standard hyperbolic plane $U(=$ an even unimodular indefinite lattice of rank 2) and two copies of the lattice $E_{8}$ (= an even unimodular negative definite lattice of rank 8). The lattice $L$ is called the K3-lattice. The Picard group $\operatorname{Pic}(X)$ of divisor 
classes of $X$ is naturally identified with the sublattice of algebraic cycles of $H_{2}(X, \mathbf{Z})$. The unimodularity of $H_{2}(X, \mathbf{Z})$ (= Poincaré duality) allows one to identify $H_{2}(X, \mathbf{Z})$ with the second cohomology group $H^{2}(X, \mathbf{Z})=\operatorname{Hom}\left(H_{2}(X, \mathbf{Z}), \mathbf{Z}\right)$ equipped with the lattice structure by means of the cup-product. Let

$$
c: \operatorname{Pic}(X) \rightarrow H^{2}(X, \mathbf{Z})
$$

be the corresponding injection. If one uses the interpretation of $\operatorname{Pic}(X)$ as the group of isomorphism classes of line bundles on $X$, the map $c$ corresponds to the first Chern class map. In virtue of the Hodge Index theorem, the lattice $\operatorname{Pic}(X)$ is of signature $\left(t_{+}, t_{-}\right)=$ $(1, t)$.

The complex structure on $X$ defines the Hodge decomposition

$$
H^{2}(X, \mathbf{C})=H^{2,0}(X) \oplus H^{1,1}(X) \oplus H^{0,2}(X)
$$

where $H^{p, q}(X) \cong H^{q}\left(X, \Omega_{X}^{p}\right)$. It is known that complex conjugation sends $H^{2,0}(X)$ to $H^{0,2}(X)$ and

$$
P_{X}=\left(H^{2,0}(X) \oplus H^{0,2}(X)\right) \cap H^{2}(X, \mathbf{R})
$$

is a positive definite 2-plane in $H^{2}(X, \mathbf{R})$. The subspace

$$
H_{\mathbf{R}}^{1,1}(X)=H^{1,1}(X) \cap H^{2}(X, \mathbf{R})
$$

has signature $(1,19)$. The cone

$$
V(X)=\left\{x \in H_{\mathbf{R}}^{1,1}(X) \cap H^{2}(X, \mathbf{R}):(x, x)>0\right\}
$$

consists of two connected components. We denote by $V(X)^{+}$the component which contains the class of some Kähler form on $X$ with respect to the complex structure of $X$. Let

$$
\Delta(X)=\{\delta \in \operatorname{Pic}(X):(\delta, \delta)=-2\}
$$

By Riemann-Roch, $\Delta(X)=\Delta(X)^{+} \coprod \Delta(X)^{-}$where $\Delta(X)^{+}$consists of effective classes and $\Delta(X)^{-}=-\Delta(X)^{+}$. Let $W(X)$ be the subgroup of the orthogonal group of $H^{2}(X, \mathbf{Z})$ generated by reflections in elements from $\Delta(X)$. This group acts properly discontinuously in $V(X)^{+}$with fundamental domain

$$
C(X)=\left\{x \in V(X)^{+}:(x, \delta) \geq 0, \quad \text { for any } \delta \in \Delta(X)^{+}\right\}
$$

The set $C(X)^{+}$of its interior points is the the Kähler cone of $X$ ([13], Exposé $\left.\mathrm{X}\right)$.

By the Lefschetz Theorem

$$
\operatorname{Pic}(X)=H_{\mathbf{R}}^{1,1}(X) \cap H^{2}(X, \mathbf{Z})
$$

We set

$$
\operatorname{Pic}(X)^{+}=C(X) \cap H^{2}(X, \mathbf{Z}), \quad \operatorname{Pic}(X)^{++}=C(X)^{+} \cap H^{2}(X, \mathbf{Z}) .
$$


The elements of $\operatorname{Pic}(X)^{+}$are pseudo-ample divisor classes, i.e., numerically effective divisor classes with positive self-intersection. Elements of $\operatorname{Pic}(X)^{++}$are ample divisor classes. Elements from $V(X)^{+} \cap H^{2}(X, \mathbf{Z})$ are just effective divisor classes with positive selfintersection.

Now let $M$ be an even non-degenerate lattice of signature $(1, t)$. The cone

$$
V(M)=\left\{x \in M_{\mathbf{R}}:(x, x)>0\right\} \subset M_{\mathbf{R}}
$$

consists of two connected components. We fix one of them and denote it by $V(M)^{+}$.

Let

$$
\Delta(M)=\{\delta \in M:(\delta, \delta)=-2\} .
$$

We fix a subset $\Delta(M)^{+}$such that

(i) $\Delta(M)=\Delta(M)^{+} \amalg \Delta(M)^{-}$, where $\Delta(M)^{-}=\left\{-\delta: \delta \in \Delta(M)^{+}\right\}$;

(ii) if $\delta_{1}, \ldots, \delta_{k} \in \Delta(M)^{+}$and $\delta=\sum n_{i} \delta_{i}$ with $n_{i} \geq 0$ then $\delta \in \Delta(M)^{+}$.

The choice of subset $\Delta(M)^{+}$as above defines the subset

$$
C(M)^{+}=\left\{h \in V(M)^{+} \cap M:(h, \delta)>0 \quad \text { for all } \delta \in \Delta(M)^{+}\right\} .
$$

Definition. An $M$-polarized $\mathrm{K} 3$ surface is a pair $(X, j)$ where $X$ is a $\mathrm{K} 3$ surface and $j: M \hookrightarrow \operatorname{Pic}(X)$ is a primitive lattice embedding. We say that $(X, j)$ is pseudo-ample (resp. ample) $M$-polarized if

$$
j\left(C(M)^{+}\right) \cap \operatorname{Pic}(X)^{+} \neq \emptyset
$$

(resp.

$$
\left.j\left(C(M)^{+}\right) \cap \operatorname{Pic}(X)^{++} \neq \emptyset\right) .
$$

Two $M$-polarized $\mathrm{K} 3$ surfaces $(X, j)$ and $\left(X^{\prime}, j^{\prime}\right)$ are called isomorphic if there exists an isomorphism of K3 surfaces $f: X^{\prime} \rightarrow X$ such that $j=f^{*} \circ j^{\prime}$.

Remarks (1.1). Note that for any pseudo-ample $M$-polarized K3 surface $(X, j)$ we have

(i) $j\left(V(M)^{+}\right) \subset V(X)^{+}$;

(ii) $j\left(\Delta(M)^{+}\right)=j(M) \cap \Delta(X)^{+}$.

Conversely, if these conditions are satisfied, and $j(M)=\operatorname{Pic}(X)$, then $(X, j)$ is ample $M$-polarized.

Finally observe that a pseudo-ample $M$-polarized K3 surface is algebraic.

Example (1.2). Let $M=<2 n>:=\mathbf{Z} e,(e, e)=2 n$. Assume $n>0$. Choose $V(M)^{+}$ to be one of the two rays in $M_{\mathbf{R}} \backslash\{0\}$. A pseudo-ample $M$-polarized $\mathrm{K} 3$ surface $(X, j)$ is called a degree $2 n$ pseudo-polarized $K 3$ surface. Consider the complete linear system $|j(e)|$ and let $f$ be a rational map defined by this linear system. Then one of the following three possible cases occurs:

(i) (Unigonal case) $|j(e)|$ has a base curve $C \cong \mathbf{P}^{1},|j(e)-C|=|(n+1) E|$ where $E$ is an elliptic curve. The map $f$ is a regular map from $X$ to $\mathbf{P}^{n+1}$ whose image is a normal rational curve of degree $n+1$. 
(ii) (Hyperelliptic case) $|j(e)|$ has no base points and $f$ is a morphism of degree 2 onto a normal surface of degree $n$ in $\mathbf{P}^{n+1}$. Its singular points (if any) are rational double points.

(iii) $|j(e)|$ has no base points and $f$ is a morphism of degree 1 onto a normal surface of degree $2 n$ in $\mathbf{P}^{n+1}$. Its singular points (if any) are rational double points.

Moreover, if $j(e)$ is ample, the unigonal case may occur only if $n=1$. Also in cases (ii) and (iii), the morphism $f$ is finite and its image is nonsingular (see [13], Exposé IV).

2. Local deformations. Fix an $M$-polarized $K 3$ surface $\left(X_{0}, j_{0}\right)$. Let $S$ be the local moduli space for $X_{0}$. It is smooth of dimension 20 with all Kodaira-Spencer mappings

$$
\rho_{s}: T_{s}(S) \rightarrow H^{1}\left(X_{s}, \Theta_{X_{s}}\right)
$$

being isomorphisms. Let

$$
H^{1}\left(X_{0}, \Theta_{X_{0}}\right) \otimes H^{1}\left(X_{0}, \Omega_{X_{0}}^{1}\right) \rightarrow H^{2}\left(X_{0}, \mathcal{O}_{X_{0}}\right)
$$

be the natural pairing induced by the duality map $\Theta_{X_{0}} \otimes \Omega_{X_{0}}^{1} \rightarrow \mathcal{O}_{X_{0}}$. As was explained in the previous section, we can view $\operatorname{Pic}(X)$ as a subgroup of $H^{1,1}\left(X_{0}\right)$. We denote by

$$
H^{1}\left(X_{0}, \Theta_{X_{0}}\right)_{j_{0}}
$$

the orthogonal complement of $j_{0}(M)$ with respect to the pairing (1). Let

$$
H^{1}\left(X_{0}, \Omega_{X_{0}}^{1}\right)_{j_{0}}=H^{1}\left(X_{0}, \Omega_{X_{0}}^{1}\right) / j_{0}(M) \text {. }
$$

In view of the canonical pairing

$$
H^{1,1}\left(X_{0}\right) \otimes H^{1,1}\left(X_{0}\right) \rightarrow H^{2,2}\left(X_{0}\right) \cong \mathbf{C}
$$

we may identify $H^{1}\left(X_{0}, \Omega_{X_{0}}^{1}\right)_{j_{0}}$ with

$$
H^{1,1}\left(X_{0}\right)_{j_{0}}:=\left(j_{0}(M)_{\mathbf{C}}\right)_{H^{1,1}\left(X_{0}\right)}^{\perp} .
$$

The pairing (1) induces the map

$$
d_{1}: H^{1}\left(X_{0}, \Theta_{X_{0}}\right)_{j_{0}} \rightarrow \operatorname{Hom}\left(H^{1}\left(X_{0}, \Omega_{X_{0}}^{1}\right)_{j_{0}}, H^{2}\left(X_{0}, \mathcal{O}_{X_{0}}\right)\right)
$$

Proposition (2.1). There is a local moduli space $S_{M}$ of isomorphism classes of $M$ polarized K3 surfaces. It is smooth of dimension $19-t$. Its tangent space at each point $s \in S$ is naturally isomorphic to $H^{1}\left(X_{0}, \Theta_{X_{0}}\right)_{j_{0}}$.

Proof. In the case $t=0$ this is a theorem from [15]. The general case is proved similarly. 
Let

$$
H^{1}\left(X_{0}, \Theta_{X_{0}}\right) \otimes H^{0}\left(X_{0}, \Omega_{X_{0}}^{2}\right) \rightarrow H^{1}\left(X_{0}, \Omega_{X_{0}}^{1}\right)
$$

be the natural pairing induced by the contraction map $\Theta_{X_{0}} \otimes \Omega_{X_{0}}^{2} \rightarrow \Omega_{X_{0}}^{1}$. Composing (2) with the projection $H^{1}\left(X_{0}, \Omega_{X_{0}}^{1}\right) \rightarrow H^{1}\left(X_{0}, \Omega_{X_{0}}^{1}\right)_{j_{0}}$ and restricting the composition to $H^{1}\left(X_{0}, \Theta_{X_{0}}\right)_{j_{0}}$ we get the map

$$
d_{2}: H^{1}\left(X_{0}, \Theta_{X_{0}}\right)_{j_{0}} \rightarrow \operatorname{Hom}\left(H^{0}\left(X_{0}, \Omega_{X_{0}}^{2}\right), H^{1}\left(X_{0}, \Omega_{X_{0}}^{1}\right)_{j_{0}}\right)
$$

Let

$$
\left(d_{1}, d_{2}\right): H^{1}\left(X_{0}, \Theta_{X_{0}}\right)_{j_{0}} \rightarrow \operatorname{Hom}\left(H^{1,1}\left(X_{0}\right)_{j_{0}}, H^{0,2}\left(X_{0}\right)\right) \oplus \operatorname{Hom}\left(H^{2,0}\left(X_{0}\right), H^{1,1}\left(X_{0}\right)_{j_{0}}\right)
$$

The formula

$$
\left(\theta_{1}, \theta_{2}\right)=d_{1}\left(\theta_{2}\right) \circ d_{2}\left(\theta_{1}\right): H^{2,0}\left(X_{0}\right) \rightarrow H^{0,2}\left(X_{0}\right)
$$

defines the bilinear form

$$
H^{1}\left(X_{0}, \Theta_{X_{0}}\right)_{j_{0}}^{\otimes 2} \rightarrow \operatorname{Hom}\left(H^{2,0}\left(X_{0}\right), H^{0,2}\left(X_{0}\right)\right)
$$

The canonical pairing

$$
H^{2,0}\left(X_{0}\right) \otimes H^{0,2}\left(X_{0}\right) \rightarrow H^{2,2}\left(X_{0}\right)=H^{4}\left(X_{0}, \mathbf{C}\right) \cong \mathbf{C}
$$

allows one to identify the space of values of the bilinear form with the space $H^{0,2}\left(X_{0}\right)^{\otimes 2}$. One can check that this pairing is symmetric [16] and hence defines the linear map

$$
\mathrm{Yu}: S^{2}\left(H^{1}\left(X_{0}, \Theta_{X_{0}}\right)_{j_{0}}\right) \rightarrow H^{0,2}\left(X_{0}\right)^{\otimes 2}
$$

which we call the Griffiths-Yukawa quadratic map for $M$-polarized K3 surfaces. A choice of an isomorphism

$$
H^{0,2}\left(X_{0}\right) \cong \mathbf{C}
$$

allows one to identify the map Yu with a quadratic form on the space $H^{1}\left(X_{0}, \Theta_{X_{0}}\right)_{j_{0}}$.

The Griffiths-Yukawa quadratic map can also be expressed in terms of the intersection form on $H^{1,1}\left(X_{0}\right)$ as follows. First observe that the map

$$
d_{1}: H^{1}\left(X_{0}, \Theta_{X_{0}}\right)_{j_{0}} \rightarrow \operatorname{Hom}\left(H^{2,0}\left(X_{0}\right), H^{1,1}\left(X_{0}\right)_{j_{0}}\right) \cong H^{0,2}\left(X_{0}\right) \otimes H^{1,1}\left(X_{0}\right)_{j_{0}}
$$

is bijective (since it is injective and both spaces have the same dimension). The pairing

$$
H^{1,1}\left(X_{0}\right)_{j_{0}} \otimes H^{1,1}\left(X_{0}\right)_{j_{0}} \rightarrow \mathbf{C}
$$

defines the symmetric bilinear map

$$
\left(H^{2,0}\left(X_{0}\right)^{*} \otimes H^{1,1}\left(X_{0}\right)_{j_{0}}\right) \otimes\left(H^{2,0}\left(X_{0}\right)^{*} \otimes H^{1,1}\left(X_{0}\right)_{j_{0}}\right) \rightarrow H^{0,2}\left(X_{0}\right)^{\otimes 2} .
$$


Then it follows from the definition of the Griffiths-Yukawa quadratic map that

$$
\mathrm{Yu}=\mathrm{Yu}^{\prime} \circ d_{1},
$$

where

$$
\mathrm{Yu}^{\prime}: H^{2,0}\left(X_{0}\right) \otimes H^{1,1}\left(X_{0}\right)_{j_{0}} \rightarrow H^{0,2}\left(X_{0}\right)^{\otimes 2}
$$

is the quadratic map defined by (4).

3. The period map. The map (3) can be naturally interpreted as the differential of the period mapping for $M$-polarized K3 surfaces. Let $M$ be a lattice of signature $(1, t)$ embeddable into the K3-lattice $L$. Fix a lattice embedding $i_{M}: M \rightarrow L$. We shall often identify $M$ with the image $i_{M}(M)$. Let

$$
N=M_{L}^{\perp}
$$

be the orthogonal complement of $M$ in $L$. It is a lattice of signature $(2,19-t)$.

Definition. A marked $M$-polarized $K 3$ surface is a pair $(X, \phi)$, where $X$ is a K3 surface together with an isomorphism of lattices $\phi: H^{2}(X, \mathbf{Z}) \rightarrow L$ such that $\phi^{-1}(M) \subset \operatorname{Pic}(X)$. The pair $\left(X, j_{\phi}\right)$ with $j_{\phi}=\phi^{-1} \mid M: M \rightarrow \operatorname{Pic}(X)$ is a $M$-polarized K3 surface. In this way we can speak about pseudo-ample and ample marked $M$-polarized K3 surfaces. Two marked surfaces $(X, \phi)$ and $\left(X^{\prime}, \phi^{\prime}\right)$ are called isomorphic if there exists an isomorphism of surfaces $f: X \rightarrow X^{\prime}$ such that $\phi^{\prime}=\phi \circ f^{*}$.

Given a marked $M$-polarized K3 surface $(X, \phi)$, the Hodge decomposition of $H^{2}(X, \mathbf{C})$ defines the point $\phi\left(H^{2,0}(X)\right)$ in $\mathbf{P}\left(L_{\mathbf{C}}\right)$. Since $H^{2,0}(X)$ is orthogonal to $H^{1,1}(X)$ (with respect to the cup-product in $\left.H^{2}\right)$, the line $\phi\left(H^{2,0}\right)$ is always orthogonal to $\phi(j(M))=M$. Therefore

$$
\phi\left(H^{2,0}(X)\right) \in \mathbf{P}\left(N_{\mathbf{C}}\right) \subset \mathbf{P}\left(L_{\mathbf{C}}\right) .
$$

Let $Q$ be the quadric in $\mathbf{P}\left(N_{\mathbf{C}}\right)$ corresponding to the quadratic form on $N_{\mathbf{C}}$ defined by the lattice $N$. For any $\omega \in H^{2,0}(X)$ we have

$$
(\omega, \omega) \in H^{4,0}(X)=\{0\} .
$$

This shows that $\phi\left(H^{2,0}\right) \in Q$. Also

$$
(\omega, \bar{\omega}) \in \mathbf{R}_{+} \subset H^{2,2}(X) \cap H^{4}(X, \mathbf{R})=\mathbf{R} .
$$

Therefore $\phi\left(H^{2,0}(X)\right)$ is contained in an open (in the usual topology) subset $D_{M}$ of the quadric $Q$ defined by the inequality $(\omega, \bar{\omega})>0$. By assigning to $H^{2,0}\left(X_{0}\right)$ the positive definite 2-plane $P_{X} \subset N_{\mathbf{R}}$ together with the orientation defined by the choice of the isotropic line $H^{2,0}\left(X_{0}\right) \subset P_{X} \otimes \mathbf{C}$, we can identify $D_{M}$ with the symmetric homogeneous space $O(2,19-t) / S O(2) \times O(19-t)$ of oriented positive definite 2 -planes in $N_{\mathbf{R}}$. The space consists of two connected components each isomorphic to a bounded Hermitian domain of 
type $I V_{19-t}$. The involution which switches the two components is induced by the complex conjugation map $Q \rightarrow Q$. We shall call the point $\phi\left(H^{2,0}\right) \in D_{M}$ the period point of $(X, \phi)$.

Let $S_{M}$ be the local moduli space of an $M$-polarized K3 surface $\left(X_{0}, j_{0}\right)$. Since $S_{M}$ is contractible, we can choose a marking $\phi: H^{2}\left(X_{s}, \mathbf{C}\right) \rightarrow L$ for all $X_{s}, s \in S_{M}$. We fix this marking and identify $H^{2}\left(X_{s}, \mathbf{C}\right)$ with $L_{\mathbf{C}}$. The complex structure on $X_{s}$ defines the Hodge decomposition and hence the period point $H^{2,0}\left(X_{s}\right) \in D_{M}$. By the Local Torelli Theorem ([13], Exposé V) the resulting period map

$$
p: S_{M} \rightarrow D_{M}
$$

is a holomorphic map which is locally an isomorphism in a neighborhood of the origin $0=\left(X_{0}, j_{0}\right)$. Let $\mu=p(0) \in D_{M}$ be the period point of $\left(X_{0}, j_{0}\right)$. We shall identify it with the subspace $H^{2,0}$ of $N_{\mathbf{C}}$. Then

$$
T_{\mu}\left(D_{M}\right) \cong H o m\left(\mu, \mu^{\perp} / \mu\right)=H o m\left(H^{2,0}\left(X_{0}\right), H^{1,1}\left(X_{0}\right)_{j_{0}}\right) \cong H^{0,2}\left(X_{0}\right) \otimes H^{1,1}\left(X_{0}\right)_{j_{0}} .
$$

The differential of the period map

$$
d p_{0}: T_{0}\left(S_{M}\right) \rightarrow T_{\mu}\left(D_{M}\right)
$$

is the bijective map $d_{1}$ from $(3)$.

Let $\mathcal{K}_{M}$ be the fine moduli space of marked $M$-polarized K3 surfaces. It is obtained by gluing local moduli spaces of marked $M$-polarized K3 surfaces and is a (non-separated) analytic space (see [13], Exposé XIII, [30]). The local period maps are glued together to give a holomorphic map

$$
p: \mathcal{K}_{M} \rightarrow D_{M}
$$

This map is the restriction of the period map $P: \mathcal{M} \rightarrow D \supset D_{M}$ for marked Kähler K3 surfaces. According to the Global Torelli Theorem of Burns-Rappoport and the Surjectivity Theorem of Todorov the latter map is étale and surjective (see loc.cit). The former theorem also describes the fibres of the period map.

Let $(X, \phi)$ be a marked $M$-polarized K3 surface. Then the image of the data

$$
\left(P_{X}, V(X)^{+}, \Delta(X)^{+}, C(X)\right)
$$

under the map $\phi$ defines the data $\left(\pi, V_{\pi}^{+}, \Delta_{\pi}^{+}, C_{\pi}\right)$, where

(i) $\pi$ is a positive oriented 2-plane in $N_{\mathbf{R}}$;

(ii) $V_{\pi}^{+}$is a connected component of the cone $\left\{x \in \pi^{\perp}:(x, x)>0\right\}$;

(iii) $\Delta_{\pi}^{+}$is a subset of $\Delta_{\pi}=\left\{e \in \pi^{\perp} \cap L:(e, e)=-2\right\}$ such that $\Delta_{\pi}=\Delta_{\pi}^{+} \amalg-\Delta_{\pi}^{+}$;

(iv) $C_{\pi}=\left\{x \in V_{\pi}^{+}:(x, e) \geq 0\right.$ for any $\left.e \in \Delta_{\pi}^{+}\right\}$.

Note that $V_{\pi}^{+}$is uniquely determined by $\pi$ (since $V(M)^{+}$is fixed) and $C_{\pi}$ is determined by $\Delta_{\pi}^{+}$.

Theorem (3.1). The restriction of the period map $p: \mathcal{K}_{M} \rightarrow D_{M}$ to the subset $\mathcal{K}_{M}^{p a}$ of isomorphism classes of marked pseudo-ample $M$-polarized K3 surfaces is surjective. There is a natural bijection between the fibre of the map

$$
p^{\prime}: \mathcal{K}_{M}^{p a} \rightarrow D_{M}
$$


over a point $\pi \in D_{M}$ and the subgroup $W_{\pi}(N)$ of isometries of $L$ generated by reflections in vectors from $\Delta_{\pi} \cap N$.

Proof. It follows from the Global Torelli Theorem that the fibre $p^{-1}(\pi)$ is bijective to the set of possible pairs $\left(V_{\pi}^{+}, \Delta_{\pi}^{+}\right)$. The group $W_{\pi}(L) \times\{ \pm 1\}$, where $W_{\pi}(L)$ is generated by reflections in elements from $\Delta_{\pi}$, acts transitively on the fibre. Pick up a point $(X, \phi)$ in $p^{-1}(\pi)$ corresponding to $\left(V_{\pi}^{+}, \Delta_{\pi}^{+}\right)$such that $V(M)^{+} \subset V_{\pi}^{+}$. Let $h \in C(M)^{+}$, we may choose $(X, \phi)$ such that $h \in C_{\pi}$. This is possible because $C_{\pi}$ is a fundamental domain for the action of $W_{\pi}(L)$ in $V_{\pi}^{+}$. Since $\pi \in D_{M}, \phi^{-1}(M) \subset \operatorname{Pic}(X)$ and $j_{\phi}(h) \in \operatorname{Pic}(X)^{+}$. Composing $\phi$ with some reflections from $\Delta(M)$, we may assume that $h \in j_{\phi}\left(C(M)^{+}\right)$. Thus $(X, \phi)$ is a marked pseudo-ample $M$-polarized K3 surface with $p((X, \phi))=\pi$. This proves the surjectivity.

Let $(X, \phi) \in \mathcal{K}_{M}^{p a}$ then the fibre of $p^{\prime}: \mathcal{K}_{M}^{p m} \rightarrow D_{M}$ over $\pi=p((X, \phi))$ is bijective to the set of subsets $\Delta_{\pi}^{+}$such that $\Delta_{\pi}^{+} \cap M=C(M)^{+}$. The stabilizer $G$ of this set in $W_{\pi}(L)$ is the subgroup $W_{\pi}(N)$. In fact, it follows from the properties of reflection groups that $G$ is generated by reflections in vectors $\delta \in \Delta_{\pi}$ such that $(\delta, h)=0$, where $h \in C(M)^{+}$. Since $C(M)^{+}$linearly spans $M_{\mathbf{R}}$ we must have $\delta \in N$. This proves the theorem.

For any $\delta \in \Delta(N)=\{x \in N:(x, x)=-2\}$, set

$$
\begin{gathered}
H_{\delta}=\left\{z \in N_{\mathbf{C}}:(z, \delta)=0\right\}, \\
D_{M}^{\circ}=D_{M} \backslash\left(\bigcup_{\delta \in \Delta(N)} H_{\delta} \cap D_{M}\right) .
\end{gathered}
$$

Let $(X, \phi)$ be an ample marked $M$-polarized K3 surface. Then $\left(j_{\phi}(M)\right)^{\perp} \cap H^{1,1}$ cannot contain vectors $v$ with $(v, v)=-2$. This shows that the period point $\pi=\phi\left(P_{X}\right)$ satisfies $\Delta_{\pi} \cap N=\emptyset$. This implies the following:

Corollary (3.2). Let $\mathcal{K}_{M}^{a}$ denote the subset of $\mathcal{K}_{M}$ which consists of isomorphism classes of marked ample $M$-polarized $K 3$ surfaces. The period map induces a bijective map

$$
p: \mathcal{K}_{M}^{a} \stackrel{\sim}{\rightarrow} D_{M}^{\circ}
$$

Next we want to get rid of markings of $M$-polarized K3 surfaces. For any lattice $S$ we denote by $O(S)$ its orthogonal group. The group

$$
\Gamma(M)=\{\sigma \in O(L): \sigma(m)=m \quad \text { for any } m \in M\} .
$$

acts on the moduli space $\mathcal{K}_{M}$ transforming $(X, \phi)$ to $(X, \phi \circ \sigma)$ without changing the isomorphism class of the $M$-polarized K3 surface $\left(X, j_{\phi}\right)$.

Let $\Gamma_{M}$ be the image of $\Gamma(M)$ under the natural injective homomorphism

$$
\Gamma(M) \rightarrow O(N)
$$


Proposition (3.3). Let $A(N)=N^{*} / N$ be the discriminant group of the lattice $N$, and let $O(N) \rightarrow \operatorname{Aut}(A(N))$ be the natural homomorphism. Denote its kernel by $O(N)^{*}$. Then

$$
\Gamma_{M}=O(N)^{*} .
$$

In particular, $\Gamma_{M}$ is a subgroup of finite index in $O(N)$.

Proof. This follows from Corollary 1.5.2 in [31].

The group $O(N)$ is an arithmetic subgroup of $O(2,19-t)$ and by the previous proposition so is $\Gamma_{M}$. Since $D_{M}$ is the union of two copies of a Hermitian homogeneous domain, the quotient $D_{M} / \Gamma_{M}$ is a quasi-projective algebraic variety with at most two irreducible components. Obviously $\Gamma(M)$ contains the subgroup of $O(L)$ generated by reflections in vectors $\delta \in N$ with $(\delta, \delta)=-2$. Thus each fibre of the map $p^{\prime}: \mathcal{K}_{M}^{p a} \rightarrow D_{M}$ is mapped to the same orbit in $\mathbf{K}_{M} / \Gamma(M)$. Applying Theorem 3.1, we obtain that the period mapping descends to a bijection

$$
\mathcal{K}_{M}^{p a} / \Gamma(M) \cong D_{M} / \Gamma_{M} .
$$

Since the elements of the quotient set $\mathcal{K}_{M}^{p a} / \Gamma(M)$ are isomorphism classes of pseudo-ample $M$-polarized $\mathrm{K} 3$ surfaces, we are able to endow the set $\mathcal{K}_{M}^{p a} / \Gamma(M)$ with a structure of a quasi-projective algebraic variety. We denote this variety by $\mathbf{K}_{M}$.

Assume that $M$ satisfies the following condition:

(U) For any two primitive embeddings $i_{1}, i_{2}: M \hookrightarrow L$, there exists an isometry $\sigma: L \rightarrow L$ such that $i_{1} \circ \sigma=i_{2}$.

Let $(X, j)$ be a pseudo-ample $M$-polarized K3 surface. Take any marking $\phi: H^{2}(X, \mathbf{Z})$ $\rightarrow L$. Composing it with $j: M \rightarrow H^{2}(X, \mathbf{Z})$, we obtain a primitive embedding $i^{\prime}: M \rightarrow$ $L$. Replacing $\phi$ with $\phi \circ \sigma$ for appropriate isometry $\sigma$ of $L$, we obtain a new marking $\phi^{\prime}: H^{2}(X, \mathbf{Z}) \rightarrow L$ such that $j=j_{\phi^{\prime}}$. This shows that any isomorphism class of $(X, j)$ is represented by a point of $\mathbf{K}_{M}$. So we may view $\mathbf{K}_{M}$ as the moduli space of $M$-polarized K3 surfaces.

Similarly we can define the variety $\mathbf{K}_{M}^{a}$ of isomorphism classes of ample $M$-polarized K3 surfaces. We have

$$
\mathbf{K}_{M}^{a}=D_{M}^{\circ} / \Gamma_{M} .
$$

Observe that $O(N)$ has only finitely many orbits in the set of primitive vectors with given value of the quadratic form (for example, this follows from Proposition 1.15.1 of [31]). This shows that the complement of $D_{M}^{\circ} / \Gamma_{M}$ in $D_{M} / \Gamma_{M}$ is the union of finitely many hypersurfaces, in particular, $D_{M}^{\circ} / \Gamma_{M}$ is an open Zariski subset of $D(M) / \Gamma_{M}$.

Remark (3.4). In fact, following [13], éxpose XIII, one can show that $D_{M} / \Gamma_{M}$ is a coarse moduli space of pseudo-ample $M$-polarized K3 surfaces. First we define a family of $M$-polarized K3 surfaces. This is a family $f: \mathcal{X} \rightarrow S$ of K3 surfaces together with a homomorphism of sheaves $M_{S} \rightarrow \mathcal{P} i c_{\mathcal{X} / S}$ where $\mathcal{P} i c_{\mathcal{X} / S} \subset R^{2} f_{*}(\mathbf{Z})$ is the relative Picard sheaf. We can define a family of pseudo-ample $M$-polarized K3 surfaces by requiring additionally that each $\left(f^{-1}(s), j_{s}\right)$ is pseudo-ample $M$-polarized K3 surface. Since $\mathcal{K}_{M}$ is a fine moduli space for marked $M$-polarized K3 surfaces, a family $(f: \mathcal{X} \rightarrow S, \phi)$ of marked $M$-polarized K3 surfaces is equivalent to a holomorphic map $\alpha: S \rightarrow \mathcal{K}_{M}$. 
Composing this map with the period we obtain that $(f: \mathcal{X} \rightarrow S, \phi)$ defines a holomorphic map $\bar{\alpha}: S \rightarrow D_{M} / \Gamma_{M}$. Given a family $(f: \mathcal{X} \rightarrow S, j)$ of pseudo-ample $M$-polarized K3 surfaces, after localizing $S$, we equip it with marking, and define the map $\bar{\alpha}: S \rightarrow D_{M} / \Gamma_{M}$ which does not depend on the choice of the marking. When $S$ is a point, we get a a bijection $\mathcal{K}_{M}^{p a} / \Gamma(M) \cong D_{M} / \Gamma_{M}$. This proves that $D_{M} / \Gamma_{M}$ is a coarse moduli space. Similarly we prove that $D_{M}^{\circ} / \Gamma_{M}$ is a coarse moduli space for ample $M$-polarized K3 surfaces.

I don't know any algebraic construction for $\mathbf{K}_{M}$ except when $M$ is of rank 1.

4. Tube domain realization of the period space. Let $b: V \times V \rightarrow \mathbf{C}$ be a non-degenerate symmetric bilinear form on a complex vector space and let $Q: b(x, x)=0$ be the corresponding non-degenerate quadric in the projective space $\mathbf{P}(V)$ associated to $V$. For any non-zero vector $v \in V$ we denote by $[v]$ the line $\mathbf{C} v \in \mathbf{P}(V)$. For any $v \in V \backslash\{0\}$ the hyperplane $H_{v}=\{w \in V: b(w, v)=0\}$ intersects $Q$ along the quadric

$$
Q(v)=Q \cap H_{v}=\left\{x \in Q: v \in P T_{x}(Q)\right\} \subset H_{v},
$$

where $P T_{x}(Q)$ is the projective tangent space of $Q$ at the point $[v]$. If $b(v, v)=0$, i.e. $[v] \in Q$, the hyperplane $H_{v}$ coincides with $P T_{[v]}(Q)$ and $Q(v)$ is the cone over the quadric $\bar{Q}(v) \subset \mathbf{P}\left(H_{v} / \mathbf{C} v\right)$ with the vertex at $[v]$. In other words, the projection map $Q \backslash\{[v]\} \rightarrow$ $\mathbf{P}(V / \mathbf{C} v)$ is an isomorphism outside $Q(v)$, and blows down $Q(v) \backslash\{[v]\}$ to the quadric $\bar{Q}(v)$.

We shall apply the previous remarks to our situation where $V=N_{\mathbf{C}} \subset L_{\mathbf{C}}$ with the symmetric bilinear form defined by the lattice $N=M^{\perp}$. The period space $D_{M}$ is a subset of the quadric $Q$ defined by the inequality $(\mu, \bar{\mu})>0$.

Lemma (4.1). Let $f \in\left(M^{\perp}\right)_{\mathbf{R}},(f, f)=0$. Then

$$
D_{M} \cap Q([f])=\emptyset .
$$

Proof. Suppose there exists $\mu \in D_{M} \cap Q([f])$. Since $f$ is a real vector, and the bilinear form originates from the lattice structure, we have $(\bar{\mu}, f)=(\mu, f)=0$. This implies that $f \in P^{\perp}$, where $P \subset N_{\mathbf{R}}$ is the positive definite 2-plane spanned by the real and imaginary part of $\mu$. However, the signature $\left(t_{+}, t_{-}\right)$of the space $\left(M^{\perp}\right)_{\mathbf{R}}$ satisfies $t_{+}=2$. Therefore $P^{\perp}$ is negative definite and does not contain isotropic vectors. This contradiction proves the assertion.

From now on we assume that $t \leq 18$, i.e., $\operatorname{rank}(M) \leq 19$. This ensures that the lattice $N=M^{\perp}$ is indefinite. Let us fix an isotropic vector $f \in N_{\mathbf{R}}$. We set

$$
\begin{gathered}
W_{f}=\left\{x \in N_{\mathbf{R}}:(x, f)=1\right\} / \mathbf{R} f, \\
V_{f}=\left\{x \in N_{\mathbf{R}}:(x, f)=0\right\} / \mathbf{R} f .
\end{gathered}
$$

By Lemma (4.1), the projection map $\pi: Q \backslash Q([f]) \rightarrow \mathbf{P}\left(N_{\mathbf{C}} / \mathbf{C} f\right)$ maps $D_{M}$ isomorphically onto a subset of the affine space

$$
A_{f}=\mathbf{P}\left(N_{\mathbf{C}} / \mathbf{C} f\right) \backslash \mathbf{P}\left(\left(V_{f}\right)_{\mathbf{C}}\right) \cong\left\{z \in N_{\mathbf{C}}:(z, f)=1\right\} / \mathbf{C} f=
$$




$$
W_{f}+i V_{f}=\left\{z=x+i y \in N_{\mathbf{C}} / \mathbf{C} f=\left(N_{\mathbf{R}} / \mathbf{R} f\right)+i\left(N_{\mathbf{R}} / \mathbf{R} f\right):(x, f)=1,(y, f)=0\right\} .
$$

Theorem (4.2). The projection map $Q \backslash Q([f]) \rightarrow \mathbf{P}\left(N_{\mathbf{C}} / \mathbf{C} f\right)$ defines an analytic isomorphism

$$
D_{M} \cong\left\{x+i y \in A_{f}:(y, y)>0\right\} .
$$

Proof. This is just the translation of the condition $(\mu, \bar{\mu})>0$ in terms of the projection map. We write any $\mu \in D_{M}$ in the form $\mu=\lambda f+x+i y$ where $x+\mathbf{R} f \in W_{f}, y+\mathbf{R} f \in V_{f}$. We have

$$
0=(\mu, \mu)=[2 \Re(\lambda)+(x, x)-(y, y)]+i[2 \Im(\lambda)+2(x, y)],
$$

hence $(x, x)-(y, y)+2 \Re(\lambda)=0$. This implies that

$$
0<(\mu, \bar{\mu})=2 \Re(\lambda)+(x, x)+(y, y)=2(y, y) .
$$

This proves the assertion.

Recall that for any real affine space $W$ with the translation space $V$ and an open connected cone $C \subset V$ which does not contain an affine line, the set

$$
\Omega(W, V, C)=\{z=x+i y: x \in W, y \in C\} \subset W_{\mathbf{C}}
$$

is called the tube domain associated to the cone $C$ in $V$. In the special case when $V$ is equipped with a nondegenerate quadratic form with signature $(1, n)$ and the cone $V^{+}$ is one of the two connected components of the cone $\{x \in V:(x, x)>0\}$ the tube domain $\Omega(W, V, C)$ is a bounded Hermitian symmetric domain of type $I V_{n}$. This can be applied to our situation where $V=V_{f}$. Fix a connected component $V_{f}^{+}$of the cone $\left\{x \in V_{f}:(x, x)>0\right\}$. Restricting the period map to a connected component $D_{M}^{+}$of $D_{M}$, we obtain

Corollary (4.3). The choice of an isotropic vector $f \in N_{\mathbf{R}}$ defines an isomorphism

$$
D_{M}^{+} \cong \Omega\left(W_{f}, V_{f}, V_{f}^{+}\right)
$$

Corollary (4.4). For any $\mu \in D_{M}$ the choice of a representative $\ell \in L$ of $\mu$ with $(\ell, f)=1$ defines a canonical isomorphism

$$
\alpha_{\mu}: T_{\mu}\left(D_{M}\right) \rightarrow\left(V_{f}\right)_{\mathbf{C}}
$$

If $[(X, \phi)] \in \mathcal{K}_{M}$ is the isomorphism class of a marked $M$-polarized $K 3$ surface with the period point $\mu$, then the pre-image of the quadratic form on $\left(V_{f}\right)_{\mathbf{C}}$ under the map

$$
\alpha_{\mu} \circ d p_{[(X, \phi)]}: H^{1}\left(X, \Theta_{X}\right) \rightarrow\left(V_{f}\right)_{\mathbf{C}}
$$

coincides with the Griffiths-Yukawa quadratic form with respect to the normalization $H^{0,2} \stackrel{\sim}{\rightarrow} \mathbf{C}$ defined by the linear function $\phi^{-1}(\ell) \in H^{2,0}$.

Proof. The map

$$
\alpha_{\mu}: T_{\mu}\left(D_{M}\right)=\operatorname{Hom}\left(\mu, \mu^{\perp} / \mu\right) \rightarrow\left(V_{f}\right)_{\mathbf{C}}
$$


is the composition of the differential of the map $D_{M} \rightarrow A_{f}$ at the point $\mu$ and the differential of the translation map $A_{f} \rightarrow\left(V_{f}\right)_{\mathbf{C}}, z \mapsto z-\mu$. Explicitly it sends $\psi: \mu \rightarrow \mu^{\perp} / \mu$ to $\psi(\ell)^{\prime}-\left(\psi(\ell)^{\prime}, f\right) \ell \bmod \mathbf{C} f$, where $\psi(\ell)^{\prime}$ is a representative of $\psi(\ell)$ in $\mu^{\perp}$.

Remark (4.5). In general there is no canonical trivialization of the affine space $W_{f}$. However, a choice of an isotropic vector $g \in N_{\mathbf{R}}$ with $(f, g)=1$ defines the trivialization

$$
W_{f} \rightarrow\left(V_{f}\right), x \rightarrow x-g
$$

If we choose to identify $V_{f}$ with $(\mathbf{R} f+\mathbf{R} g)_{N_{\mathbf{R}}}^{\perp}$, then the explicit isomorphism $\alpha: \Omega_{f} \rightarrow D_{M}$ is given by the formula:

$$
\alpha(z)=\mathbf{C}\left(-\frac{1}{2}(z, z) f+g+z\right)
$$

5. Some arithmetical conditions on $M$. We are going to put some arithmetical conditions on our lattice $M$ to ensure, for example, condition (U) in section (3).

For each non-degenerate even lattice $S$ we denote by $A(S)=S^{*} / S$ the discriminant group of $S$ equipped with the quadratic map

$$
q_{S}: A(S) \rightarrow \mathbf{Q} / 2 \mathbf{Z}, \quad q_{M}(x+S)=(x, x)+2 \mathbf{Z},
$$

where the bilinear form of $S$ is extended to a $\mathbf{Q}$-valued bilinear form on $S^{*}$.

For example, for any integer $m \neq 0$, let $U(m)$ denote the lattice of rank 2 with a basis $\left(e, e^{\prime}\right)$ such that $\left(e, e^{\prime}\right)=m,(e, e)=\left(e^{\prime}, e^{\prime}\right)=0$. Then $A(U(m))=(\mathbf{Z} / m \mathbf{Z})^{2}$ with $q_{U(m)}$ defined by the formula:

$$
q((a+m \mathbf{Z}, b+m \mathbf{Z}))=\frac{2 a b}{m}+2 \mathbf{Z} .
$$

It is clear that for any isometry $\sigma \in O(L)$, we have a canonical isomorphism $D_{M} \rightarrow$ $D_{\sigma(M)}$ which defines a canonical isomorphism of the moduli spaces

$$
\mathcal{K}_{M} \cong \mathcal{K}_{\sigma(M)}
$$

where we choose $\left(V(M)^{+}, \Delta(\sigma(M))^{+}\right)$to be equal to $\left(\sigma\left(V(M)^{+}\right), \sigma\left(\Delta(M)^{+}\right)\right.$. The next result of Nikulin gives a condition implying that any two primitive embeddings $i: M \rightarrow$ $L, i^{\prime}: M \rightarrow L$ differ by an isometry of $L$.

Proposition (5.1). Let $S$ be an even lattice of signature $(1, t)$ with $t \leq 19$. Assume that $l(A(S)) \leq 20-t$ or $t \leq 10$. Then there exists a primitive embedding $S \hookrightarrow L$. Moreover, such an embedding is unique up to an isometry of $L$ if for each prime $p \neq 2$ the p-primary component $A(S)_{p}$ of $A(S)$ satisfies $l\left(A(S)_{p}\right) \leq 19-t$ and, if $l\left(A(S)_{2}=21-t\right.$, $A(S)_{2}$ contains as a direct summand the discriminant form of the lattice $U(2)$.

Proof. See [31], Corollary 1.12.3, Theorems 1.12.4, 1.14.4. 
Corollary (5.2). Any even lattice $M$ of signature $(1, t)$ with $t \leq 9$ admits a unique primitive embedding in the K3-lattice $L$. In particular, the moduli space $\mathbf{K}_{M}^{a}$ of ample $M$-polarized K3 surfaces is not empty (and of dimension $19-t$ ).

Proof. In fact, $\mathbf{K}_{M}^{a}$ is a Zarisli-open non-empty subset in (19-t)-dimensional algebraic variety $D_{M} / \Gamma_{M}$.

Next we want to study primitive isotropic vectors $f$ in a non-degenerate even lattice $S$. Consider $f$ as an element of $S^{*}$ and let $\operatorname{div}(f)$ be the positive generator of the image of the linear map $f: S \rightarrow \mathbf{Z}$. Let $f^{*}=\frac{1}{\operatorname{div}(f)} f+S \in S^{*}$. Clearly $f^{*}$ is an isotropic element of the discriminant quadratic form $A(S)$. Let $I(S)$ denote the set of primitive isotropic vectors in $S$, and $I(A(S))$ be the same for $A(S)$. The map $f \rightarrow f^{*}+S$ defines a map $I(S) \rightarrow I(A(S))$. The orthogonal group $O(S)$ acts naturally on the source and the target of this map, and the map is compatible with this action. Let

$$
O(S)^{*}=\operatorname{Ker}(O(S) \rightarrow O(A(S)) .
$$

This group acts on the fibres of the map $I(S) \rightarrow I(A(S))$.

Proposition (5.3). The map

$$
I(S) / O(S)^{*} \rightarrow I(A(S)), f \mapsto f^{*}+S
$$

is surjective if $S$ admits the lattice $U=U(1)$ as an orthogonal summand. The map is bijective if $S$ admits the lattice $U \perp U$ as an orthogonal summand.

Proof. See [37], Lemmas 4.1.1 and 4.1.2.

Definition. An isotropic vector $f \in I(S)$ is called $m$-admissible if $\operatorname{div}(f)=m$ and there exists $g \in I(S)$ with $(f, g)=m, \operatorname{div} g=m$.

Lemma (5.4). The following conditions are equivalent:

(i) $f \in I(S)$ is $m$-admissible;

(ii) there exists a primitive lattice embedding $i: U(m) \rightarrow S$ such that $S=i(U(m)) \oplus$ $i(U(m))^{\perp}$ and $f \in i(U(M))$.

Proof. $(i) \Rightarrow(i i)$. Let $g \in I(S)$ such that $(f, g)=m$. The sublattice $U^{\prime}$ spanned by $f$ and $g$ is primitive, contains $f$ and is isomorphic to $U(M)$. Since for any $s \in S, m$ divides $(s, f)$ and $(s, g)$, we obtain $s-\frac{(s, g)}{m} f-\frac{(s, f)}{m} g \in U_{S}^{\perp}$. This shows that $S=U^{\prime} \perp U^{\perp}$.

$(i) \Leftarrow(i i)$ Obvious.

Proposition (5.5). Let $S$ be an even indefinite non-degenerate lattice of signature $\left(t_{+}, t-\right)$. Then $S$ admits the lattice $U(m)$ as an orthogonal summand if the following conditions are satisfied:

(i) $A(U(m))$ is isomorphic to an orthogonal summand of $A(S)$ with respect to the bilinear form defined by $q_{S}$;

(ii) $l(A(S)) \leq t_{+}+t_{-}-3$.

Proof. Let $A^{\prime}$ be the orthogonal complement of $A(U(m))$ in $A(S)$. Then $l\left(A^{\prime}\right) \leq$ $l(A(S)) \leq \operatorname{rank} S-3<\left(t_{+}-1\right)+\left(t_{-}-1\right)$. By Corollary 1.10.2 from [31], there exists 
a lattice $S^{\prime}$ with signature $\left(t_{+}-1, t_{-}-1\right)$ and $A\left(S^{\prime}\right) \cong A^{\prime}$. Thus the lattice $U(m) \perp S^{\prime}$ has the same signature and the same discriminant quadratic form as the lattice $S$. By Corollary 1.13.3 from loc. cit. we obtain $S \cong U(m) \perp S^{\prime}$.

Proposition (5.6). Suppose $M^{\perp}$ contains an $m$-admissible isotropic vector with $m \leq 2$. Then the moduli space $\mathbf{K}_{M}$ is irreducible.

Proof. Let $M^{\perp}=U(m) \perp M^{\prime}$. The isometry $-\operatorname{id}_{U(m)} \oplus \operatorname{id}_{M^{\prime}}$ of $M^{\perp}$ acts identically on the discriminant group of $M^{\perp}$, hence extends to an isometry of $\sigma \in \Gamma(M)$ of $L$ (see Proposition (3.3)). Obviously $\sigma$ switches the orientation of a positive definite 2-plane $\pi \subset\left(M^{\perp}\right)_{\mathbf{R}}$ spanned by a vector $x \in U(m)$ with $(x, x)>0$ and a vector $y \in M^{\prime}$ with $(y, y)>0$. Hence $\sigma$ switches the two connected components of $D_{M}$. This implies that $D_{M} / \Gamma(M)=D_{M} / \Gamma_{M}$ is irreducible.

6. Mirror symmetry. Now we are ready to define the mirror family. Pick up an $m$-admissible isotropic vector $f$ in $N=M^{\perp}$ (Proposition (5.3) gives some sufficient conditions for its existence). Then $M^{\perp}=U^{\prime} \perp \dot{M}$, where $U^{\prime} \cong U(m)$ and $f \in U^{\prime}$. The sublattice $\check{M}$ is of signature $(1,18-t)$. We have

$$
(\mathbf{Z} f)_{M^{\perp}}^{\perp} / \mathbf{Z} f \cong \check{M} .
$$

So as an abstract lattice, $\check{M}$ does not depend on the choice of $U^{\prime} \cong U(m)$ containing $f$. Let us fix $\left(V(\check{M})^{+}, \Delta(\check{M})^{+}, C(\check{M})^{+}\right)$and use the embedding $i: \check{M} \hookrightarrow M^{\perp} \subset L$ to introduce the moduli space $\mathbf{K}_{\check{M}}$ of $\check{M}$-polarized K3 surfaces.

Definition. The moduli space $\mathbf{K}_{\check{M}}$ is called the mirror moduli space of $\mathbf{K}_{\check{M}}$.

The definition depends obviously on the choice of $U^{\prime}$ which determines the embedding $\check{M} \hookrightarrow L$. If we replace $i$ with composition $i^{\prime}=\sigma \circ i$ where $\sigma \in O(L)$, then $i^{\prime}(M) \subset$ $\sigma\left(M^{\perp}\right)=\sigma(M)^{\perp}$. Thus the new $\mathbf{K}_{i^{\prime}(\check{M})}$ will be equal to the mirror of $\mathbf{K}_{\sigma(M)} \cong \mathbf{K}_{M}$. Thus, if we put conditions on $M$ which ensure the uniqueness of primitive embedding of $\check{M}$ in $L$, we obtain that the isomorphism class of the mirror moduli space depends only on the choice of $f \in M^{\perp}$. Since the signature and the discriminant group of $\check{M}$ can be read off from the signature and discriminant of $M$, we can apply Proposition (5.1) to get some sufficient conditions on $M$ which guarantee that our construction is well-defined.

Note the obvious relations

$$
\begin{gathered}
\operatorname{dim} \mathbf{K}_{\check{M}}+\operatorname{dim} \mathbf{K}_{M}=20, \\
\operatorname{dim} \mathbf{K}_{\check{M}}=\operatorname{rank} M=\operatorname{rank} \operatorname{Pic}(X),
\end{gathered}
$$

where for any marking $\phi$ of $X \in \mathbf{K}_{M}$ the period of $(X, \phi)$ does not belong to a subvariety of the form $D_{M^{\prime}}$ for some sublattice $M^{\prime}$ of $L$ with $M \subset M^{\prime}$.

Note that $\mathbf{K}_{M}$ is not a fine moduli space, so there is no a universal family of pseudoample $M$-polarized K3 surfaces. We shall usually substitute it with a family $f: \mathcal{X} \rightarrow S$ of pseudo-ample $M$-polarized K3 surfaces (in sense of Reamrk (3.4)) such that the period 
map $S \rightarrow \mathbf{K}_{M}$ is of finite degree. A similar family $f^{\prime}: \mathcal{X}^{\prime} \rightarrow S^{\prime}$ of $\check{M}$-polarized K3 surfaces will be called a mirror family.

The mirror correspondence works especially nicely when $m=1$. This is true if and only if $M^{\perp}$ contains an isotropic vector with $\operatorname{div}(f)=1$. Choose $U^{\prime} \cong U$ containing $f$. Then $M^{\perp}=U^{\prime} \perp \check{M}$ and $\check{M} \perp=U^{\prime} \perp M$. Thus, we can use $f \in U^{\prime}$ to define the mirror family for both $\mathbf{K}_{M}$ and $\mathbf{K}_{\check{M}}$. Since

$$
\check{\check{M}}=M
$$

we obtain that the mirror correspondence is a duality. Note that additional assumptions on $M$ guarantee that this duality is independent of the choice of $U^{\prime}$. For example, suppose $l\left(A(M) \leq t-3\right.$. Then $A(\check{M}) \cong A\left(M^{\perp}\right) \cong A(M)$ and by Corollary 1.13.3 of [31] $\check{M}$ is determined uniquely by its signature and the discriminant form. Proposition 1.15.1 of loc. cit. implies that the $U$-splitting of $M^{\perp}$ is unique up to an isometry of $M^{\perp}$. Applying Proposition (5.1) to $M^{\perp}$ we obtain that $M^{\perp}$ admits a unique primitive embedding in $L$. Thus any isometry of $M^{\perp}$ lifts to an isometry of $L$. This shows that the moduli space $\mathbf{K}_{\check{M}}$ is independent of the choice of splitting $M^{\perp}=U \perp \check{M}$.

Remark (6.1). One of the main motivation of Nikulin's paper [31] was to find some conditions ensuring that two lattices $S$ and $S^{\prime}$ are K3-dual, i.e., can be realized as the lattices $M$ and $\check{M}$ from above. For example, he proves in [31] (Corollary 1.13.5) that two hyperbolic lattices $S$ and $S^{\prime}$ are K3-dual if rank $S+\operatorname{rank} S^{\prime}=20$ and $A(S) \cong A\left(S^{\prime}\right)$ as abelian groups and the values of the discriminant quadratic forms differ by sign. The notion of K3-dual lattices plays an important rôle in the explanation of the Arnold's Strange Duality where $M$ occurs as the lattice generated by algebraic cycles supported at infinity for a K3-smoothing of one of the fourteen unimodal exceptional singularities and $\check{M} \perp U$ is realized as the Milnor lattice of vanishing cycles for the same singularity. The Strange Duality switches the role of the lattices $M$ and $\check{M}$. In [31] Nikulin proves that the Milnor lattice of a hypersurface surface singularity contains an 1-admissible isotropic vector whenever it is indefinite.

For any K3 surface $X$ we can introduce the tube domain (the Picard tube domain)

$$
\operatorname{Ptd}(X)=\operatorname{Pic}(X)_{\mathbf{R}}+i C(X)^{+}
$$

Now let $(X, j)$ be an $M$-polarized K3 surface and $f \in M^{\perp}$ be an $m$-admissible isotropic vector. Fix a splitting $M^{\perp}=U^{\prime} \perp \check{M}$ where $U^{\prime} \cong U(m)$ and $f \in U^{\prime}$. Let us consider the tube domain $\Omega_{f}=V_{f}+i V_{f}^{+}$. Observe that

$$
V_{f}=\left((\mathbf{Z} f)_{M^{\perp}}^{\perp} / \mathbf{Z} f\right)_{\mathbf{R}} \cong \check{M}_{\mathbf{R}} \subset L_{\mathbf{R}}
$$

Let us choose the component $V_{f}^{+}$such that under the above isomorphism

$$
V_{f}^{+}=V(\check{M})^{+}
$$


Let

$$
\begin{gathered}
V_{f}^{++}=C(\check{M})_{\mathbf{R}}^{+}=\left\{y \in V(\check{M})^{+}:(y, \delta)>0 \quad \text { for all } \delta \in \Delta(\check{M})\right\} \\
\Omega_{f}^{+}=V_{f}+i V_{f}^{++}=\check{M}_{\mathbf{R}}+C(\check{M})_{\mathbf{R}}^{+} .
\end{gathered}
$$

Let $(X, \phi)$ be an ample $\check{M}$-polarized surface. Then

$$
\Delta(\check{M})^{+}=j_{\phi}^{-1}\left(\Delta(X)^{+}\right)
$$

The map $j_{\phi}: \check{M} \rightarrow \operatorname{Pic}(X)$ defines an open subset

$$
V_{f}^{++}(X, \phi)=j_{\phi}^{-1}\left(C(X)^{+}\right)
$$

of $V_{f}^{++}$, and a holomorphic embedding

$$
V_{f}+i V_{f}^{++}(X, \phi) \hookrightarrow \operatorname{Ptd}(X) .
$$

Note that, if $j_{\phi}$ is an isomorphism we get $V_{f}^{++}(X, \phi)=V_{f}^{++}$and the previous embedding becomes an isomorphism.

Let $g \in U_{\mathbf{R}}^{\prime}$ be an isotropic vector with $(f, g)=1$. By Remark (4.5), it defines an isomorphism from each connected component of $D_{M}$ onto the tube domain $\Omega_{f}$. Let $D_{M}^{+}$ be the pre-image of $\Omega_{f}^{+}$under this isomorphism and let $\mathcal{K}_{M}^{+}$be the pre-image of $D_{M}^{+}$under the period map from Theorem (4.2). For any ample $\check{M}$-polarized marked K3 surface $(X, \phi)$ with bijective $j_{\phi}: \check{M} \rightarrow \operatorname{Pic}(X)$, the period map defines a holomorphic isomorphism:

$$
\mathcal{K}_{M}^{+} \cong \operatorname{Ptd}(X)
$$

Note that its definition depends on the choice of splitting $M^{\perp}=U^{\prime} \perp \check{M}$, the choice of an isotropic vector $f \in U(m)$, and the choice of marking $\phi$.

Recall that the period space $D_{M}$ admits a compactification $D_{M}^{*}$ which is isomorphic to the quadric in $\mathbf{P}\left(N_{\mathbf{C}}\right)$ defined by the lattice $N$. The topological boundary of $D_{M}$ in $D_{M}^{*}$ is equal to the disjoint union of locally closed analytic subsets $F$, called the boundary components. Each boundary component is of the form $\mathbf{P}\left(I_{\mathbf{C}}\right) \cap \overline{D_{M}}$ for some isotropic subspace $I$ of $N_{\mathbf{R}}$. Since $N$ is of signature $(2,19-t)$, we have either $\operatorname{dim} I=1(F$ is a point) or $\operatorname{dim} I=2$ ( $F$ is isomorphic to upper half plane). The stabilizer group $N(F)=\left\{g \in O\left(N_{\mathbf{R}}\right): g(F)=F\right\}$ of $F$ is a maximal parabolic subgroup of $G=O\left(N_{\mathbf{R}}\right)$. Conversely, each such subgroup occurs as $N(F)$ for some boundary component $F$. A boundary component $F$ is called rational if the corresponding isotropic subspace can be defined over $\mathbf{Q}$. It is clear that we can identify the set of isotropic subspaces of $N_{\mathbf{Q}}$ with the set of primitive isotropic sublattices of $N$. In particular we have a bijective correspondence

$\left\{0\right.$-dimensional rational boundary components of $\left.D_{M}\right\} \longleftrightarrow I(N)$.

Let $\Gamma \subset G(\mathbf{Q})=O\left(N_{\mathbf{Q}}\right)$ be an arithmetic subgroup of $G$ (e.g., a subgroup of finite index in $O\left(N_{\mathbf{Z}}\right)$ ). It acts on the set $\mathcal{R B}\left(D_{M}\right)$ of rational boundary components of $D_{M}$, 
and for each such component $F$, the stabilizer group $N_{\Gamma}(F)=\Gamma \cap N(F)$ acts discretely on $F$ with algebraic quotient $F / N_{\Gamma}(F)$. Same is true for $D / \Gamma$. We have

$$
\overline{D_{M} / \Gamma}=D / \Gamma \coprod\left(\bigcup_{F \in \mathcal{R} \mathcal{B}\left(D_{M}\right)} F\right) / \Gamma=D / \Gamma \coprod\left(\bigcup_{F \in \mathcal{R} \mathcal{B}\left(D_{M}\right) / \Gamma} F / N_{\Gamma}(F)\right)
$$

is a normal projective algebraic variety (Baily-Borel-Satake compactification).

We shall apply it to our situation when $\Gamma=\Gamma_{M}$. Let $f \in I(N)$ and let $F$ be the corresponding zero-dimensional rational boundary component of $D_{M}$. We set

$$
Z_{M}(f)=\left\{g \in N_{\Gamma_{M}}(F): g(f)=f\right\}
$$

Now let us assume that $f \in I(N)$ is $m$-admissible and fix a splitting $M^{\perp}=U^{\prime} \perp \check{M}$ where $U^{\prime} \cong U(m), f \in U^{\prime}$. Let $g \in U^{\prime}$ be an isotropic vector with $(g, f)=m$.

Proposition (6.2). Let $O(\check{M})^{*}=\operatorname{Ker}(O(\check{M}) \rightarrow O(A(\check{M}))$. Then there is a canonical split extension of groups

$$
0 \rightarrow m \check{M} \rightarrow Z_{M}(f) \rightarrow O(\check{M})^{*} \rightarrow 1
$$

Proof. We can write any $n \in M^{\perp}$ in the form $n=a f+b g+z$, where $a, b \in \mathbf{Z}, z \in \check{M}$. Any $\sigma \in Z_{M}(f)$ is defined by the formula

$$
\sigma(f)=f, \quad \sigma(g)=-\frac{\left(v_{\sigma}, v_{\sigma}\right)}{2 m} f+g+v_{\sigma}, \quad \sigma(z)=-\frac{\left(v_{\sigma}, \tilde{\sigma}(z)\right)}{m} f+\tilde{\sigma}(z),
$$

for some $v_{\sigma}, \tilde{\sigma}(z) \in \check{M}$. It is easy to check that $\tilde{\sigma}: z \rightarrow \tilde{\sigma}(z)$ is an element of $O(\check{M})$. Setting $A(\sigma)=\left(\tilde{\sigma}, v_{\sigma}\right)$ we verify that

$$
A\left(\sigma^{\prime} \circ \sigma\right)=\left(\tilde{\sigma}^{\prime} \circ \tilde{\sigma}, \tilde{\sigma}^{\prime}\left(v_{\sigma}\right)+v_{\sigma^{\prime}}\right)
$$

Let $G$ be the group of pairs $(s, v) \in O(\check{M}) \times \check{M}$ with the composition law $\left(s^{\prime}, v^{\prime}\right) \circ(s, v)=$ $\left(s^{\prime} \circ s, s^{\prime}(v)+v^{\prime}\right)$. The homomorphism $(s, v) \mapsto s$ makes it an extension of $O(\check{M})$ with help of $\check{M}$. It splits by the section $s \mapsto(s, 0)$. The homomorphism $\sigma \mapsto A(\sigma)$ is an injective homomorphism from $Z_{M}(f)$ to $G$. To find its image we have to decide which pairs $\left(\tilde{\sigma}, v_{\sigma}\right)$ correspond to isometries $\sigma \in O\left(M^{\perp}\right)$ which lift to isometries from $\Gamma(M)$. By Proposition (3.3), the condition for this is that $\sigma \in \operatorname{Ker}\left(O\left(M^{\perp}\right) \rightarrow O\left(A\left(M^{\perp}\right)\right)\right.$. It is easy to check that any $\sigma$ with $A(\sigma)=\left(1, v_{\sigma}\right)$ satisfies this condition if and only if $\frac{v_{\sigma}}{m} \in \check{M}$. Each $\sigma$ with $A(\sigma)=(\tilde{\sigma}, 0)$ satifies this condition if and only if $\tilde{\sigma} \in O(\check{M})^{*}$. Since $G$ is the semi-direct product of $\check{M}$ and $O(\check{M})$, we get that the image of $Z_{M}(f)$ is the semi-diect product of $m \check{M}$ and $O(\check{M})^{*}$. This proves the lemma.

Let $Z_{M}(f)^{+}$be the subgroup of $Z_{M}(f)$ whose image in $O(\check{M})^{*}$ consists of elements preserving $C(\check{M})^{+}$. The group $Z_{M}(f)$ acts naturally on $\Omega_{f}=\check{M}_{\mathbf{R}}+i \check{M}_{\mathbf{R}}^{+}$by the formula

$$
\left(\tilde{\sigma}, v_{\sigma}\right)(x+i y)=x+v_{\sigma}+i \tilde{\sigma}(y)
$$


The subgroup $Z_{M}(f)^{+}$preserves the tube domain $\Omega_{f}^{+}=V_{f}+i V_{f}^{++}=\check{M}_{\mathbf{R}}+i C(\check{M})_{\mathbf{R}}^{+}$. It follows from the theory of compactification of homogeneous symmetric domains that there exists a $N_{\Gamma}(F)$-invariant neighborhood $\tilde{U}^{*}$ of $F$ in $D_{M}^{*}$ such that the map $\tilde{U}^{*} / N_{\Gamma}(F) \rightarrow$ $D_{M}^{*} / \Gamma$ is an analytic isomorphism to a neighborhood $U^{*}$ of the boundary point $F / N_{\Gamma}(F)$ of $\overline{D_{M} / \Gamma}$. Restricting this isomorphism to $\tilde{U}=\tilde{U}^{*} \cap \Omega_{f}^{+}$we obtain an isomorphism

$$
\alpha: \tilde{U} / Z_{M}(f)^{+} \rightarrow U_{F} \subset U_{F}^{*} .
$$

The multi-valued map

$$
\alpha^{-1}: U_{F} \rightarrow \tilde{U}_{F} \subset \Omega_{f}^{+} \cong \operatorname{Ptd}\left(X^{\prime}\right)
$$

with the monodromy group $Z_{M}(f)^{+}$is the mirror map MS4' mentioned in the introduction.

Remark (6.3). By the Global Torelli Theorem for algebraic K3 surfaces the group $Z_{M}(f)^{+} / \check{M}$ contains a subgroup of finite index isomorphic to the automorphism group of any $\operatorname{surface}$ with $\operatorname{Pic}(X) \cong \check{M}$.

Let $(X, j)$ be a $M$-polarized K3 surface such that its isomorphism class $[(X, j)]$ belongs to the open subset $U_{F}$ from above. The pre-image of $U_{F}$ in $D_{M}$ is equal to the disjoint union of $\Gamma$-translates of $\tilde{U}_{F}$. So, we can choose a marking $\phi$ of $(X, j)$ such that the period point $P(X, \phi)$ belongs to $\tilde{U}_{F}$. Let $\omega$ be a holomorphic 2 -form on $X$, the function

$$
\psi([(X, j)])=\int_{\phi^{-1}(f)} \omega
$$

is a single-valued holomorphic function on $U_{F}$ (because the 2-cycle $\phi^{-1}(f)$ does not depend on the marking modulo the action of the group $\left.Z_{M}(f)^{+}\right)$. Thus if we normalize $\omega$ by replacing it with $\omega^{\prime}=\omega / \int_{\phi^{-1}(f)} \omega$, we will be able to choose a representative $\ell$ of $P(X, \phi)$ with the property $(\ell, f)=1$. By Corollary (4.4) to Theorem (4.2), we obtain that this normalization allows us to identify the Griffiths-Yukawa quadratic form on $H^{1}\left(X, \Theta_{X}\right)$ with the complex quadratic form $\check{M}_{\mathbf{C}}$. In particular, it gives an integral structure on $H^{1}\left(X, \Theta_{X}\right)$ compatible with the quadratic forms. This gives MS3' from introduction.

7. Mirrors for the family of degree $2 n$ polarized K3 surfaces. Here we consider the mirror construction in the case $M=<2 n>$. It is known ([17], Theorem 1.1.2) that $M$ admits a primitive embedding in $L$, which is unique modulo $O(L)$. Since the lattice $U$ represents any even integer, we may assume that $M \subset U$ where $L=U^{\perp 3} \perp E_{8}^{\perp 2}$. This immediately implies that $M^{\perp} \cong U \perp U \perp E_{8} \perp E_{8} \perp<-2 n>$. Write

$$
n=\tilde{n} k^{2},
$$

where $\tilde{n}$ is square free. Then the group $\Gamma_{M}$ has exactly $\left[\frac{k+2}{2}\right]$ orbits in the set of primitive isotropic vectors in the lattice $M^{\perp}$ (see [37], Theorem 4.01). Each orbit is represented by a vector $f$ with $\operatorname{div} f=d, d \mid k$, and

$$
(\mathbf{Z} f)^{\perp} / \mathbf{Z} f \cong U \perp E_{8} \perp E_{8} \perp<-2 N>:=M_{N},
$$


where $N=n / d$. So we have $\left[\frac{k+2}{2}\right]$ mirror families, each one is isomorphic to $\mathbf{K}_{M_{n / d}}$ for some $d \mid k$. Since the lattice $M_{N}$ admits a unique embedding into $L$ up to isometry of $L$, the number of non-isomorphic mirror moduli spaces for $\mathbf{K}_{M}$ is equal to the number of divisors of $k$. To study the mirror moduli spaces we may assume that $d=1$ by replacing $n$ with $n^{\prime}=n / k$. The corresponding isotropic vector can be taken from a copy of $U$. The mirror family $\mathbf{K}_{\check{M}}$ is one-dimensional and is isomorphic to $D_{M_{n}} / \Gamma_{M_{n}}$. We have $\left(M_{n}\right)^{\perp}=U \perp<2 n>$. So if we choose a standard basis $(f, g)$ of $U$, then we can find a representative $\mu$ of a point from $D_{M_{n}}^{+}$in the form

$$
\mu=-n t^{2} f+g+t e, t \in \mathbf{C} .
$$

The map $\mu \rightarrow t$ defines an isomorphism from $D_{M_{n}}^{+}$to $\Omega_{f}^{+}$. The latter can be identified with the upper half-plane $H=\{t=x+i y \in \mathbf{C}: y>0\}$. The group $Z_{\Gamma\left(M_{n}\right)}(F)^{+}$is isomorphic to $\left\langle 2 n>\cong \mathbf{Z}\right.$. Let $T$ be a generator of $Z_{\Gamma\left(M_{n}\right)}(F)^{+}$corresponding to the generator $e$ of $<2 n>$. Then $T(\check{m})=\check{m}$ for $\check{m} \in \check{M}=M_{n}, T(f)=f, T(g)=g-n f+e, T(e)=e-2 n f$ (see the proof of Proposition (6.2)). Then $T^{-1}(e)=e+2 n f$ and

$$
(\mu, f)=1, \frac{1}{2 n}(\mu, e)=t
$$

From this it follows that $T$ acts on $H$ by the formula $T(t)=t-1$. We can choose the open set $\tilde{U}_{F}$ to be equal to $\{t=x+i y: y>r\}$ for sufficiently large $r$. The map $t \rightarrow q=e^{2 \pi i t}$ defines an isomorphism

$$
\tilde{U}_{F} / Z_{\Gamma\left(M_{n}\right)}(F)^{+} \cong U_{F}=\left\{q \in \mathbf{C}^{*}:|q| \leq 1 / r\right\}
$$

Choose any marking $\phi: H_{2}(X, \mathbf{Z}) \rightarrow L$ of a $M_{n}$-polarized K3 surface $(X, j) \in U_{F}$ such that the period $\mathbf{C} \phi(\omega)$ of $(X, \phi)$ belongs to $D_{M_{n}}^{+}$and is equal to $\mathbf{C}\left(-n t^{2} f+g+t e\right)$. Then

$$
t=\frac{\frac{1}{2 n} \int_{\phi^{-1}(e)} \omega}{\int_{\phi^{-1}(f)} \omega}
$$

This is analogous to the situation in mirror symmetry for quintic 3-folds (see [26]).

Let us now compute the global monodromy group $\Gamma_{M_{n}}$. Let

$$
\Gamma_{0}(n)=\left\{\left(\begin{array}{ll}
a & b \\
c & d
\end{array}\right) \in S L(2, \mathbf{Z}): n \mid c\right\} /( \pm 1) \subset \Gamma=P S L(2, \mathbf{Z}) .
$$

The element

$$
F=\left(\begin{array}{cc}
0 & -1 / \sqrt{n} \\
\sqrt{n} & 0
\end{array}\right) \in P S L(2, \mathbf{R})
$$

is of order 2 and belongs to the normalizer of $\Gamma_{0}(n)$ in $P S L(2, \mathbf{R})$. It is called the Fricke involution. If we add it to $\Gamma_{0}(2)$ we obtain a subgroup of $P S L(2, \mathbf{R})$ denoted by $\Gamma_{0}(n)^{+}$. It is called the Fricke modular group of level $n$. 
Theorem (7.1). Let $\Gamma_{M_{n}}^{\prime}$ be the subgroup of index 2 of $\Gamma_{M_{n}}$ which stabilizes the connected component $D_{M_{n}}^{+} \cong H$ of $D_{M_{n}}$. Up to a conjugation in $\operatorname{PSL}(2, \mathbf{R})=\operatorname{Aut}(H)$, we have

$$
\Gamma_{M_{n}}^{\prime}=\Gamma_{0}(n)^{+} .
$$

In particular,

$$
\mathbf{K}_{M_{n}} \cong H / \Gamma_{0}(n)^{+} .
$$

Proof. The group $\Gamma_{M_{n}}$ is isomorphic to the group $O(U \perp<2 n>)^{*}$. Since $-1 \notin$ $O(U \perp<2 n>)^{*}$, the canonical homomorphism $O(U \perp<2 n>)^{*} \rightarrow P S O(1,2)$ is injective. It is known that the groups $P S L(2, \mathbf{R})=\operatorname{Aut}(H)$ and $P S O(1,2)$ are isomorphic. For example, we can establish such isomorphism by considering a natural representation of $S L(2, \mathbf{R})$ in the space $E$ of binary forms $\alpha x^{2}+2 \beta \sqrt{n} x y+\gamma y^{2}$ equipped with the quadratic form $Q(\alpha, \beta, \gamma)=2\left(n \beta^{2}-\alpha \gamma\right)$ (=twice the discriminant). This allows us to view any $g=\left(\begin{array}{ll}a & b \\ c & d\end{array}\right) \in S L(2, \mathbf{R})$ as an isometry of $(U \perp<2 n>)_{\mathbf{R}}$ defined by the matrix

$$
A(g)=\left(\begin{array}{ccc}
a^{2} & -2 a b \sqrt{n} & b^{2} \\
-a c / n & a d+b c & -b d / n \\
c^{2} & -2 \sqrt{n} c d & d^{2}
\end{array}\right) \in S O(1,2) .
$$

Here the basis $\left(y^{2}, 2 \sqrt{n} x y, x^{2}\right)$ of $E$ corresponds to the basis $(f, e, g)$ of $U \perp<2 n>$. The kernel of the map $A: S L(2, \mathbf{R}) \rightarrow S O(1,2), g \rightarrow A(g)$ is equal to $\{ \pm 1\}$. The image of the map $A$ is subgroup $S O(1,2)^{\prime}$ of index 2 of $S O(1,2)$ which preserves a connected component of $D_{M_{n}}$. Note that -1 acts as the identity on $D_{M_{n}}$, so when we extend $\Gamma_{M_{n}}^{\prime}$ by adding $-1_{M_{n}^{\perp}}$ and take the intersection with $S O(1,2)$, we obtain a a subgroup $\Gamma_{M_{n}}^{\prime \prime}$ of $S O(1,2)^{\prime}$ isomorphic to $\Gamma_{M_{n}}^{\prime}$. So we may assume now that $\Gamma_{M_{n}}^{\prime}=\Gamma_{M_{n}}^{\prime \prime}$. Let $\Gamma^{\prime}$ be the pre-image of $\Gamma_{M_{n}}^{\prime}$ under the map $A$. Let us describe its elements. First of all we use that, for any matrix $g \in \Gamma^{\prime}$, the coefficients of the matrix $A(g)$ are integers.

Write

$$
a=a_{1} \sqrt{a_{2}}, b=b_{1} \sqrt{b_{2}}, \quad c=c_{1} \sqrt{c_{2}}, \quad d=d_{1} \sqrt{d_{2}},
$$

where $a_{2}, b_{2}, c_{2}, d_{2}$ are square free. We have

$$
a b \sqrt{n}=k a_{1} b_{1} \sqrt{\tilde{n} a_{2} b_{2}} \in \mathbf{Z} \Longrightarrow a_{2} b_{2} \tilde{n} \in \mathbf{Z} \Longrightarrow a_{2} b_{2}=s^{2} \tilde{n}
$$

for some integer $s$. Since $s$ divides $a_{1}^{2}, b_{1}^{2}$ and $a b \sqrt{n}$, it must divide the first row of the matrix $A(g)$. This implies that $s= \pm 1$ and hence

$$
a_{2} b_{2}=\tilde{n} .
$$

Similarly, we obtain

$$
c_{2} d_{2}=\tilde{n} .
$$

Now, in view of above, $a c / \sqrt{n} \in \mathbf{Z}$ gives $\sqrt{c_{2} / b_{2}}, \sqrt{a_{2} / d_{2}} \in \mathbf{Z}$. This implies that

$$
c_{2}=b_{2}, d_{2}=a_{2}, k\left|a_{1} c_{1}, k\right| b_{1} d_{1} .
$$


Let us rewrite the matrix $A(g)$ using the previous information.

$$
A(g)=\left(\begin{array}{ccc}
a_{1}^{2} a_{2} & -2 a_{1} a_{2} b_{1} b_{2} k & b_{1}^{2} b_{2} \\
-a_{1} c_{1} / k & a_{1} d_{1} a_{2}+b_{1} c_{1} b_{2} & -b_{1} d_{1} / k \\
c_{1}^{2} b_{2} & -2 c_{1} d_{1} c_{2} b_{2} k & d_{1}^{2} a_{2}
\end{array}\right)
$$

Next we use that the discriminant group $A(U \perp<2 n>)$ is generated by the coset of $\frac{1}{2 n} e$ modulo $U \perp<2 n>$, where $e$ generates $\left\langle 2 n>\right.$. Thus elements of $O(U \perp<2 n>)^{*}$ send $e$ to $e+n v$ for some $v \in U$. This implies that $a d+b c \equiv \pm 1 \bmod 2 n$. Assume that $a d+b c \equiv 1 \bmod 2 n$. Together with $a d-b c=1$ this implies that $n \mid b c$, and hence $k^{2} a_{2} \mid c_{1} b_{1}$. If a prime $p$ divides $a_{2}$, it must divide $c_{1}$ since $p \mid b_{1}$ implies that $p$ divides the first row of $A(g)$. On the other hand, $p \mid c_{1}$ implies that $p$ divides the third row of $A(g)$. Thus $a_{2}=1$, hence

$$
a_{2}=d_{2}=1, b_{2}=c_{2}=\tilde{n}, \quad k^{2} \mid b_{1} c_{1}
$$

Let $p$ be a prime dividing $k$. It divides $a_{1} c_{1}$ and $b_{1} d_{1}$. Assume $p \mid b_{1}$, then $p \mid c_{1}$ since otherwise $p$ divides the whole first row of $A(g)$. Conversely, if $p \mid c_{1}$ then $p \mid b_{1}$. Thus $k \mid c_{1}$ and $k \mid b_{1}$, and we get

$$
g=\left(\begin{array}{cc}
a_{1} & k b_{1}^{\prime} \sqrt{\tilde{n}} \\
k c_{1}^{\prime} \sqrt{\tilde{n}} & d_{1}
\end{array}\right)=\left(\begin{array}{cc}
a^{\prime} & b^{\prime} \sqrt{n} \\
c^{\prime} \sqrt{n} & d^{\prime}
\end{array}\right)
$$

where $a^{\prime}, b^{\prime}, c^{\prime}, d^{\prime} \in \mathbf{Z}$. If $a d+b c \equiv-1 \bmod 2 n$, we obtain similarly that

$$
g=\left(\begin{array}{cc}
a^{\prime} \sqrt{n} & b^{\prime} \\
c^{\prime} & d^{\prime} \sqrt{n}
\end{array}\right)
$$

Thus we obtain that $\Gamma^{\prime}$ is equal to the subgroup of $P S L(2, \mathbf{R}$ of matrices of form (*) and $(* *)$. Obviously matrices of form $\left(^{*}\right)$ form a subgroup of index 2 in $\Gamma^{\prime}$. The whole group is generated by this subgroup and the matrix $g_{0}=\left(\begin{array}{cc}0 & -1 \\ 1 & 0\end{array}\right)$. Now

$$
\begin{aligned}
& \left(\begin{array}{cc}
1 / \sqrt[4]{n} & 0 \\
0 & \sqrt[4]{n}
\end{array}\right) \cdot\left(\begin{array}{cc}
a^{\prime} & b^{\prime} \sqrt{n} \\
c^{\prime} \sqrt{n} & d^{\prime}
\end{array}\right) \cdot\left(\begin{array}{cc}
1 / \sqrt[4]{n} & 0 \\
0 & \sqrt[4]{n}
\end{array}\right)^{-1}=\left(\begin{array}{cc}
a^{\prime} & b^{\prime} \\
n c^{\prime} & d^{\prime}
\end{array}\right), \\
& \left(\begin{array}{cc}
1 / \sqrt[4]{n} & 0 \\
0 & \sqrt[4]{n}
\end{array}\right) \cdot\left(\begin{array}{cc}
0 & -1 \\
1 & 0
\end{array}\right) \cdot\left(\begin{array}{cc}
1 / \sqrt[4]{n} & 0 \\
0 & \sqrt[4]{n}
\end{array}\right)^{-1}=\left(\begin{array}{cc}
0 & -1 / \sqrt{n} \\
\sqrt{n} & 0
\end{array}\right) .
\end{aligned}
$$

This proves the theorem.

Remarks (7.2) 1. Let use the isomorphism $\Phi: H \rightarrow D_{M_{n}}^{+}, t \rightarrow-n t^{2} f+g+t e$. Then $g=\left(\begin{array}{ll}\alpha & \beta \\ \gamma & \delta\end{array}\right) \in S L(2, \mathbf{R})$ acts on $H$ by the Moebius transformation $t \rightarrow(\alpha t+\beta) /(\gamma t+\delta)$, and

$$
\begin{gathered}
\Phi(g(t))=-n(\alpha t+\beta)^{2} f+(\gamma t+\delta)^{2} g+(\alpha t+\beta)(\gamma t+\delta) e= \\
=-n t^{2}\left(\alpha^{2} f-\frac{\gamma^{2}}{n} g-\frac{\alpha \gamma}{n} e\right)+\left(-n \beta^{2} f+\delta^{2} g+\beta \delta e\right)+t(-2 n \alpha \beta f+2 \gamma \delta g+(\alpha \delta+\beta \gamma) e) .
\end{gathered}
$$


This shows that the transformation $\Phi \circ g \circ \Phi^{-1}$ of $D_{M_{n}}^{+}$is defined, in the basis $(f, e,-g)$ by the matrix

$$
A^{\prime}(g)=\left(\begin{array}{ccc}
\alpha^{2} & -2 n \alpha \beta & n \beta^{2} \\
-\alpha \gamma / n & \alpha \delta+\gamma \beta & \beta \delta \\
\gamma^{2} / n & -2 \gamma \delta & \delta^{2}
\end{array}\right)
$$

Now if $g \in \Gamma_{0}(n)^{+}$we observe that $A^{\prime}(g) \in \Gamma_{M_{n}}^{\prime}$. This shows that

$$
\Phi \circ \Gamma_{0}(n)^{+} \circ \Phi^{-1}=\Gamma_{M_{n}}^{\prime}
$$

2. It is known that the orthogonal group of the discriminant group of the lattice $U \perp\langle 2 n\rangle$ is isomorphic to the group $(\mathbf{Z} / 2 \mathbf{Z})^{s}$, where $s$ is the number of distinct prime divisors of $n$ ([37], Lemma 3.6.1). If $n=k^{2} \tilde{n}$ as above with $(k, \tilde{n})=1$, this group is isomorphic to $\bar{\Gamma}_{0}(n) / \Gamma_{0}(n)$ where $\bar{\Gamma}_{0}(n)$ is the abelian normalizer of $\Gamma_{0}(n)$ in $S L(2, \mathbf{R})$ (see [20], Theorem 3). Using Nikulin's results from [31], one can show that the homomorphism $O(U \perp<2 n>) \rightarrow O(A(U \perp<2 n>))$ is surjective. Since $\Phi \circ \Gamma_{0}(n) \circ \Phi^{-1}$ is equal to $\Gamma_{M_{n}} \cap S O(U \perp<2 n>)$, this easily implies that

$$
\Phi \circ \bar{\Gamma}_{0}(n) \circ \Phi^{-1}=O(U \perp<2 n>)
$$

The group $O(A(U \perp<2 n>))$ acts on $\mathbf{K}_{M_{n}}$ with kernel isomorphic to $\{ \pm 1\}$. The quotient is the moduli space of K3 surfaces admiting a pseudo-ample $M_{n}$-polarization.

Let us now find the subset $\mathbf{K}_{M_{n}}^{a} \subset H / \Gamma_{0}(n)^{+}$of isomorphism classes of ample $M_{n^{-}}$ polarized K3 surfaces.

Theorem (7.3). Let $S \subset H / \Gamma_{0}(n)^{+}$be the set of orbits of the points $\frac{c}{b}+\frac{i}{b \sqrt{n}}$, where $c \in \mathbf{Z}$ and $b \mid c n^{2}+1$. Then

$$
\mathbf{K}_{M_{n}}^{a}=H / \Gamma_{0}(n)^{+} \backslash S
$$

Moreover,

$$
\# S= \begin{cases}1 & \text { if } n \leq 4 \\ 2 h(-4 n) & \text { if } n \equiv 7 \bmod 8 \\ 4 h(-4 n) / 3 & \text { if } n \equiv 3 \bmod 8, n \geq 4 \\ h(-4 n) & \text { otherwise }\end{cases}
$$

Here $h(k)$ denotes the number of classes of primitive binary quadratic forms of discriminant $k$.

Proof. Recall from section 3 that $\mathbf{K}_{M_{n}} \backslash \mathbf{K}_{M_{n}}^{a}$ is equal to the set of $\Gamma_{M_{n}}$-orbits in $D_{M_{n}}$ of hyperplanes $H_{v}=\left\{z \in D_{M_{n}}:(z, v)=0\right\}, v \in U \perp<2 n>,(v, v)=-2$. Let us use the isomorphism $\Phi: H \rightarrow D_{M_{n}}^{+}, t \rightarrow-n t^{2} f+g+t e$. Let $v=a f+b g+c e$ with $(v, v)=2 a b+2 n c^{2}=-2$. Then $\left(-t^{2} f+g+t e, v\right)=-n b t^{2}+a+2 n c t=0$ implies

$$
t=\frac{c}{b} \pm \sqrt{\frac{c^{2}}{b^{2}}+\frac{a}{n b}}=\frac{c}{b} \pm \sqrt{\frac{n c^{2}+a b}{n b^{2}}}=\frac{c}{b}+\frac{i}{b \sqrt{n}}
$$


This proves our first assertion. Let $t \in H$ such that $\Phi(t) \in H(v)$ for some hyperplane $H(v)$ as above. Since $h(v)$ is fixed by an automorphism of order 2 corresponding to the reflection isometry $x \rightarrow x+(x, v) v$, we see that $t$ is fixed by some involution $g \in \Gamma_{0}(n)^{+}$. Let $g$ be represented by a matrix $\tilde{g}=\left(\begin{array}{ll}a & b \\ c & d\end{array}\right)$. We have either $\tilde{g}^{2}=1$ or $\tilde{g}^{2}=-1$. Since the characteristic polynomial of $\tilde{g}$ is equal to $X^{2}-(a+d) X+1$, we see that only the second case occurs, and $a+d=0$. If $g \in \Gamma_{0}(n)$, then $\tilde{g}=\left(\begin{array}{cc}a & b \\ n c & -a\end{array}\right)$ where $a, b, c, d \in \mathbf{Z}$. The fixed points $t$ of $g$ can be computed, and we find that $t=\frac{a}{c n}+\frac{i}{c n}$. This differs from points $\left(^{*}\right)$ unless $n=1$. If $n=1, \Gamma_{0}(1)^{+}=\Gamma$, and there is only one orbit of such points. If $n>1$, and $g$ is an involution from the coset $F \cdot \Gamma_{0}(n)$ of the Fricke involution $F$, we find that its fixed points look like $\left({ }^{*}\right)$. Consider the double cover $p: X_{0}(n) \rightarrow X_{0}(n)^{+}=X_{0}(n) /(T)$, where $X_{0}(n)$ (resp. $X_{0}(n)^{+}$) is a nonsingular projective model of the quotient $H / \Gamma_{0}(n)$ (resp. $H / \Gamma_{0}(n)^{+}$). We have a bijective correspondence between the ramification points of this cover and $\Gamma_{0}(n)$-orbits in $\bar{H}=H \cup\{\infty\} \cup \mathbf{Q}$ whose stabilizer belongs to the coset $F \cdot \Gamma_{0}(n)$. When $n \geq 5$ one checks that $F \cdot \Gamma_{0}(n)$ does not have parabolic elements (i.e.. elements which fixes $t \in \mathbf{Q} \cup\{\infty\}$ ) and elements of finite order greater than 2. This shows that $\# S$ is equal to the number of ramification points of the double cover $p$. This number was computed by R. Fricke in [12], and it is equal to the number which we gave in the statement of the theorem. Now, it is known that the modular curve $X_{0}(n)$ is of genus 0 when $n=2,3,4$. Thus there are only 2 ramification points. One of them is an orbit with stabilizer of order 2 contained in $\Gamma_{0}(2)$. Another one is an orbit of with stabilizer of order 2 whose generator belongs to $F \cdot \Gamma_{0}(n)$. This proves the assertion.

Assume now that the curve $X_{0}(n)^{+}=\overline{H / \Gamma_{0}(n)^{+}}$is rational. All such $n$ can be listed (see [18]) (as was observed by A. Ogg, the primes from this list are just those which divide the order of the Fisher-Griess Monster group). Let

$$
C(n)=\overline{H / \Gamma_{0}(n)^{+}} \backslash\left(H / \Gamma_{0}(n)^{+}\right)
$$

be the set of cusp points.

Proposition (7.3). Let $\phi$ be the Euler function. Then

$$
\# C(n)= \begin{cases}\frac{1}{2} \sum_{d \mid n, d>0} \phi((d, n / d)) & \text { if } n \neq 4 \\ 2 & \text { if } n=4\end{cases}
$$

Proof. The number of cusps for the modular curve $X_{0}(n)$ is equal to $\sum_{d \mid n, d>0} \phi((d, n / d))$ (see [38], Proposition 1.4.1). It is known that the Fricke involution acts on this set without fixed points if $n \neq 4$ (see [18]) and has one fixed point if $n=4$. From this the result follows.

Corollary (7.4). Let $n=p$ be a prime number, $M=<2 p>$. Assume that $X_{0}(p)^{+}$is rational. Then

$$
\mathbf{K}_{\check{M}} \cong \mathbf{A}^{1}
$$


Theorem (7.5). Assume $X_{0}(n)^{+}$is rational. There exists a unique holomorphic function (called the Hauptmodule)

$$
j_{n}: H \rightarrow \mathbf{C}
$$

satisfying the following conditions:

(i) $j_{n}$ is invariant with respect to $\Gamma_{0}(n)^{+}$;

(ii) $j_{n}$ has a Fourier expansion

$$
j_{n}(t)=q^{-1}+\sum_{m=1}^{\infty} c_{m} q^{m}, \quad q=e^{2 \pi i t}
$$

(iii) the coefficients of the Fourier expansion are all integers;

(iv) considered as a meromorphic function on $X_{0}(n)^{+}$, the function $j_{n}$ has a simple point at the cusp $\Gamma_{0}(n)^{+} \cdot \infty$ and generates the field of meromorphic functions on $X_{0}(n)^{+}$.

Proof. See [18].

Let us restrict the meromorphic function $j_{n}^{-1}$ to a neihborhood $\tilde{U}_{F}=\{t=x+i y \in H$ : $y>r\}$ for sufficiently large $r$ chosen so that $j_{n}^{-1}$ is holomorphic on $\tilde{U}_{F}$. Then the properties of $j_{n}$ assure that $j_{n}^{-1}$ defines an isomorphism from $\tilde{U}_{F} /\left(\Gamma_{0}(n)^{+}\right)_{\infty}$ to a neihborhood of the cusp $\Gamma_{0}(n)^{+} \cdot \infty$. Comparing it with the discussion in the beginning of the section, we find that the mirror map at the cusp can be given by the inverse of the Hauptmodule function $j_{n}^{-1}$. This should be compared to $[\mathbf{2 1}]$.

It is well-known that $H / \Gamma_{0}(n)$ is a coarse moduli space for the isomorphism classes of pairs $(E, A)$, where $E$ is an elliptic curve, and $A$ is a cyclic subgroup of order $\mathrm{n}$ of of $E$. The Fricke involution acts on $H / \Gamma_{0}(n)$ by sending the pair $(E, A)$ to the pair $\left(E / A, E_{n} / A\right)$. Let us give an explicit geometric relationship between the isomorphism class of a $M_{n}$-polarized K3 surface respesented by a point $z \in H / \Gamma_{0}(n)^{+}$and the isomorphism class of the pair of isogeneous elliptic curves $\left(E, E^{\prime}=E / A\right)$ represented by the same point $z$. This can be used to explain the observation of B. Lian and S. Yau that the periods of certain onedimensional families of $\mathrm{K} 3$ surfaces can be expressed as the products of periods of some family of elliptic curves (see [22]). I am grateful to Dan Burns who suggested that our K3 surfaces should be related to $\operatorname{Kummer}$ surfaces $\operatorname{Kum}\left(E \times E^{\prime}\right)$.

Theorem (7.6). Let $M=\left\langle 2 n>\right.$ and $X$ be a $M_{n}$-polarized $K 3$ surface with period $t \in H$. Let $E_{t}=\mathbf{C} / \mathbf{Z}+t \mathbf{Z}$ and $E_{t}^{\prime}=\mathbf{C} / \mathbf{Z}+(-1 / n t) \mathbf{Z}$ be the corresponding pair of isogeneous elliptic curves. Then there exists a canonical involution $\tau$ on $X$ such that $X /(\tau)$ is birationally isomorphic to the Kummer surface $E_{t} \times E_{t}^{\prime} /( \pm 1)$.

Proof. The fact that there exists an involution $\tau$ on $X$ such that $X /(\tau)$ is birationally isomorphic to some Kummer surface $A /\{ \pm 1\}$ follows from the property that $r k M_{n}=19$ [25]. As is explained in loc.cit. and in [30] such an involution corresponds to a primitive embedding $i: E_{8}(2) \rightarrow M_{n}$ (the image is the sublattice of $\tau$-antiinvariant divisor classes). Here $E_{8}(2)$ denote the lattice obtained from the lattice $E_{8}$ by multiplying its quadratic form by 2 . We define this embedding to be the canonical one: $i: E_{8}(2) \rightarrow E_{8} \perp E_{8} \perp$ $U \perp<-2 n>, x \rightarrow(x, x, 0,0)$. Then it is shown that $X /(\tau) \cong \operatorname{Kum}(A)=A /\{ \pm 1\}$, where 
$A$ is an abelian surface. Let $Y$ be a minimal nonsingular model of $\operatorname{Kum}(A)$. The rational map $\pi: X \rightarrow Y$ induces an embedding of lattices of trancendental cycles $\pi^{*}: T_{Y}(2) \rightarrow T_{X}$. It is also known [32] that $\pi^{*}\left(T_{Y}(2)\right)=2 S$ where $S \subset T_{X} \otimes \mathbf{Q}$ with $S / T_{X} \cong(\mathbf{Z} / 2 \mathbf{Z})^{\alpha} \subset$ $A\left(T_{X}\right)$. If $X$ satisfies $\operatorname{Pic}(X)=M_{n}$, then $T_{X}=U \perp<2 n>$, and it is easy to see that $T_{Y}=U(2) \perp<4 n>=T_{X}(2)$. Also, it is known that $T_{A}(2) \cong T_{Y}$ (see [4], Chapter VIII, §5). Let $p: A \rightarrow Y$ be the rational map of degree 2 defined by the canonical map $A \rightarrow \operatorname{Kum}(A)$. It follows from loc.cit. that the homomorphism $p^{*}: H^{2}(Y, \mathbf{C}) \rightarrow H^{2}(A, \mathbf{C})$ preserves the Hodge structures, i.e., $p^{*}\left(H^{2,0}(Y)\right)=H^{2,0}(A)$. The same property is true for $\pi_{\mathbf{C}}^{*}:\left(T_{Y}\right)_{\mathbf{C}} \rightarrow\left(T_{X}\right)_{\mathbf{C}}$. Thus the isomorphism $p^{*} \circ\left(\pi^{*}\right)^{-1}\left(T_{X}\right)_{\mathbf{C}} \rightarrow\left(T_{A}\right)_{\mathbf{C}}$ preserves the Hodge structures. So let us compute the period of the abelian surface $A$, knowing that the period of $X$ is equal to $\mu=-n t^{2} f+g+t e \in D_{M_{n}}^{+}$. Recall that for any complex torus $T$, we have an isomorphism of lattices $H^{2}(T, \mathbf{Z}) \cong U \perp U \perp U=U^{\perp 3}$. Fix a primitive embedding $i: U \perp<2 n>\hookrightarrow U^{\perp 3}$. To be more precise, let $\Lambda=\mathbf{Z} e_{1}+\mathbf{Z} e_{2}+\mathbf{Z} e_{3}+\mathbf{Z} e_{4}$ with a fixed isomorphism $d: \Lambda^{4} \Lambda \rightarrow \mathbf{Z}$ such that $d\left(e_{1} \wedge e_{2} \wedge e_{3} \wedge e_{4}\right)=1$. Then $\bigwedge^{2} \Lambda$ has a structure of a lattice with respect to the bilinear form $(\alpha, \beta)=d(\alpha \wedge \beta)$. Consider the following basis of $\bigwedge^{2} \Lambda$ :

$$
f_{1}=e_{1} \wedge e_{2}, g_{1}=e_{3} \wedge e_{4}, f_{2}=e_{1} \wedge e_{3}, g_{2}=e_{4} \wedge e_{2}, f_{3}=e_{1} \wedge e_{4}, g_{3}=e_{2} \wedge e_{3}
$$

Then $\mathbf{Z} f_{i}+\mathbf{Z} g_{i} \cong U$, and $\mathbf{Z} f_{i}+\mathbf{Z} g_{i}$ is orthogonal to $\mathbf{Z} f_{i}+\mathbf{Z} g_{i}$ for $i \neq j$. Our embedding $i: U \perp<2 n>\rightarrow U^{3}$ can be chosen as follows: $i(f)=f_{1}, i(g)=g_{1}, i(e)=f_{2}+n g_{2}$. For simplicity of notation we denote by $i:(U \perp<2 n>)_{\mathbf{C}} \rightarrow\left(U^{\perp 3}\right)_{\mathbf{C}}$ the extension of the embedding $i$ to the injective map of the complexified spaces. We have

$$
i(\mu)=-n t^{2} f_{1}+g_{1}+t\left(f_{2}+n g_{2}\right)=-n t^{2} e_{1} \wedge e_{2}+e_{3} \wedge e_{4}+t\left(e_{1} \wedge e_{3}+n e_{4} \wedge e_{2}\right) .
$$

We immediately verify that

$$
i(\mu)=\left(-t e_{1}+e_{4}\right) \wedge\left(n t e_{2}-e_{3}\right) .
$$

Using [13], Exposé VIII, we can interpret it as follows. Let

$$
E_{t}=\mathbf{C} / \mathbf{Z}+t \mathbf{Z}, \quad E_{t}^{\prime}=\mathbf{C} / \mathbf{Z}+(-1 / n t) \mathbf{Z}
$$

be the pair of isogeneous elliptic curves. Then under a certain marking $\phi: H^{2}\left(E_{t} \times E_{t}^{\prime}, \mathbf{Z}\right) \rightarrow$ $U^{\perp 3}, \phi\left(H^{2,0}\left(E_{t} \times E_{t}^{\prime}\right)\right)=i(\mu)$. Now the assertion follows from the Global Torelli Theorem.

Let us exhibit explicitly some mirror families of the family of polarized K3 surfaces of degree $2 n$. We shall use the notation $A_{n}, D_{n}, E_{n}$ to denote the negative definite even lattice defined by the negative of the Cartan matrix of the root system of a simple Lie algebra of type $A_{n}, D_{n}, E_{n}$, respectively. We shall use the following well-known description of the Picard lattice of an elliptic surface $f: X \rightarrow S$ with a section: 
Lemma (7.7)(Shioda-Tate). Let Pic $(X)^{\prime}$ be the subgroup of Pic $(X)$ generated by irreducible components of fibres and by a section. Then the quotient group $\operatorname{Pic}(X) / \operatorname{Pic}(X)^{\prime}$ is isomorphic to the Mordell-Weil group $M W(X / S)$ of sections of the fibration.

Proof. See [7], Proposition 5.3.4.

This lemma is applied as follows. We exhibit an elliptic fibration such that $\operatorname{Pic}(X)^{\prime}$ is a subgroup of finite index in Pic $(X)$. The lemma implies that the Mordell-Weil group is finite. Then we show that it is in fact trivial. Now it is easy to find the structure of the lattice $\operatorname{Pic}(X)^{\prime}$. Its sublattice generated by a section and a fibre is isomorphic to the lattice $U$. Its orthogonal complement is isomorphic to the sum of lattices of type $A_{n}, D_{n}, E_{n}$, each is spanned by the irreducible components of a fibre which do not intersect the chosen section.

Example (7.8) $(n=1)$. We have

$$
M_{n} \cong U \perp E_{8} \perp E_{8} \perp\langle-2\rangle .
$$

Using the previous remark, it suggests to look for a K3 surface with an elliptic fibration $f: X \rightarrow \mathbf{P}^{1}$ with a section and two reducible fibres of types $\tilde{E}_{8}$ (or $I I^{*}$ in Kodaira's notation), and one reducible fibre of type $\tilde{A}_{1}$ (Kodaira's $I_{2}$ or $I I I$ ). Since the group $F^{\natural}$ of non-singular points of a fibre $F$ of type $\tilde{E}_{8}$ is isomorphic the additive group $\mathbf{C}$, and the restriction homomorphism $\mathrm{MW}\left(X / \mathbf{P}^{1}\right) \rightarrow F^{\natural}$ is known to be injective on the torsion subgroup ([7], Proposition 5.3.4), we obtain that $\operatorname{Tors}\left(\mathrm{MW}\left(X / \mathbf{P}^{1}\right)\right)$ is trivial. Hence, if $\operatorname{Pic}(X)$ is known to be of rank 19, it must be isomorphic to $M_{n}$.

To construct such a surface $X$, we take a nonsingular plane cubic $C$ and the tangent line $L$ at its inflection point. The pencil of plane cubics spanned by $C$ and $3 L$ defines a rational map $\mathbf{P}^{2} \rightarrow \mathbf{P}^{1}$. In appropriate coordinate system we can represent the pencil in the form:

$$
\lambda\left(Y^{2} Z+X^{3}+a X Z^{2}\right)+\mu Z^{3}=0 .
$$

After resolving its nine fundamental points (infinitely near to the point $(0,1,0)$ ) we arrive at a rational elliptic surface $f: V(a) \rightarrow \mathbf{P}^{1}$. It contains a degenerate fibre of type $\tilde{E}_{8}$ corresponding to $(\lambda, \mu)=(0,1)$. The irreducible singular fibres correspond to $(\lambda, \mu)=(1, b)$ where $4 a^{3}+27 b^{2}=0$. If $a \neq 0$ we have two irreducible singular fibres with ordinary double points. If $a=0$ we have one irreducible singular fibre with a cusp singularity. Let $F_{a}(b)$ denote the fibre of $f$ corresponding to $(\lambda, \mu)=(1, b)$. Let $F_{1}=F_{a}(b)$, where $4 a^{3}+27 b^{2}=0$, be an irreducible singular fibre, and let $F_{2}=F_{a}(b+1)$. Consider the double cover $X(a, b)^{\prime}$ of $V(a)$ branched along the union $F_{1} \cup F_{2}$. After resolving its singularities we obtain a K3 surface $X(a, b)$ with two reducible fibres of type $\tilde{E}_{8}$. It has additional reducible fibres: one fibre of type $\tilde{A}_{1}$ if $F_{1}$ has a node, $F_{2}$ is nonsingular $\left(a \neq 0, b \neq-\frac{1}{2}\right)$; two fibres of type $\tilde{A}_{1}$ if $F_{1}, F_{2}$ have nodes $\left(b=-\frac{1}{2}\right)$; one fibre of type $\tilde{A}_{2}^{*}$ (Kodaira's IV) if $F_{1}$ has a cusp $(a=0)$.

We have a one-parameter family $\mathcal{X}^{\prime} \rightarrow C$ of singular surfaces $X(a, b)^{\prime}$ parametrized by the affine curve $C: 4 A^{3}+27 B^{2}=0$. The map $\mathcal{X}^{\prime} \rightarrow C$ is equivariant with respect to the natural action of the group $\mu_{3}$ of third roots of unity. Its generator $\rho=e^{2 \pi i / 3}$ acts on $C$ by $(a, b) \rightarrow(\rho a, b)$ and on $\mathcal{X}^{\prime}$ via its action on $\mathbf{P}^{2}$ by the formula $X \rightarrow \rho X, Y \rightarrow Y, Z \rightarrow Z$. After dividing $\mathcal{X}^{\prime}$ by this action, we obtain a family $\pi^{\prime}: \mathcal{Y}^{\prime}=\mathcal{X}^{\prime} / \mu_{3} \rightarrow \mathbf{A}^{1}=C / \mu_{3}$. Let 
$0 \in \mathbf{A}^{1}$ be the orbit of $(0,0) \in C$ and $1 \in \mathbf{A}^{1}$ be the orbit of $\left(a,-\frac{1}{2}\right)$. For any $t \in \mathbf{A}^{1} \backslash\{0,1\}$, the fibre $\mathcal{Y}_{t}^{\prime}=\pi^{\prime-1}(t)$ has one ordinary double point. The fibre $\mathcal{Y}_{0}^{\prime} \cong X(0,0) / \mu_{3}$, it is a rational singular surface. The fibre $\mathcal{Y}_{1} \cong X\left(a,-\frac{1}{2}\right)$. It has two ordinary double points. Let

$$
\pi: \mathcal{Y} \rightarrow \mathbf{A}^{1} \backslash\{0,1\}
$$

be the composition of $\pi^{\prime}$ and the blowing up $\mathcal{Y} \rightarrow \pi^{\prime-1}\left(\mathbf{A}^{1} \subset\{0,1\}\right.$ of the locus of singular points of the fibres $\mathcal{Y}_{t}^{\prime}, t \neq 0,1$. We have constructed a family of pseudo-ample $M_{1}$-polarized K3 surfaces. The period map for the family $\mathcal{Y} \rightarrow \mathbf{A}^{1} \backslash\{0,1\}$ defines a regular map:

$$
p: \mathbf{A}^{1} \backslash\{0,1\} \rightarrow \mathbf{K}_{M_{1}} \cong H / \Gamma \cong \mathbf{A}^{1} .
$$

One can show that the period mapping $p$ can be extended to an isomorphism $\mathbf{A}^{1} \rightarrow$ $H / \Gamma$ which sends the point 0 to the orbit of $e^{2 \pi i / 3}$ and 1 to the orbit of $i$. The first point is a period of the surface $X(0,0)$, the second point is a period of the surface $X\left(a,-\frac{1}{2}\right)$. The latter surface is non-ample $M$-polarized surface. The monodromy group of our family is generated by the local monodromies at 0,1 and $\infty$. They are isomorphic to the subgroups of $\Gamma$ which stabilize $e^{2 \pi i / 3}, i$ and $\infty$, respectively. Thus the global monodromy of our family is isomorphic to $\Gamma$.

Since not all fibres are isomorphic, the period map is not constant. Hence there exists a dense subset $U$ of $\mathbf{A}^{1} \backslash\{0,1\}$ such that the Picard number of $\mathcal{Y}_{t}, t \in U$, is equal to 19, and hence $\mathrm{Pic} \mathcal{Y}_{t} \cong M_{1}$.

Observe that $X(0,0) \cong X_{0}$ has the Picard lattice of rank 20 isomorphic to $U \perp E_{8} \perp$ $E_{8} \perp A_{2}$, and $X\left(a,-\frac{1}{2}\right)$ has Picard lattice of rank 20 isomorphic to $U \perp E_{8} \perp E_{8} \perp<$ $-2>\perp<-2>$.

Example (7.9) $(n=2)$. So we want to describe a mirror family for quartic surfaces. One can show, for example, using the uniqueness results from [31], that

$$
M_{n}=U \perp E_{8} \perp E_{8} \perp<-4>\cong U \perp E_{8} \perp D_{9} .
$$

Similar to the previous example, we should construct a one-dimensional family of elliptic K3 surfaces $X$ with a section, one reducible fibre of type $\tilde{D}_{9}$ (Kodaira's $I_{5}^{*}$ ) and one reducible fibre of type $\tilde{E}_{8}$. To construct the family $\mathcal{F}$ of such elliptic surfaces we use the same idea as in the previous example. Consider the pencil of cubic curves:

$$
F(\lambda, \mu)=\lambda X^{3}+\mu Z\left(Y^{2}-X Z+a X^{2}\right)=0 .
$$

Let $V \rightarrow \mathbf{P}^{1}$ be the associated rational elliptic surface. It has a degenerate fibre of type $\tilde{E}_{7}$ (Kodaira's $\left.I I I^{*}\right)$ and a reducible fibre $F_{1}$ of type $\tilde{A}_{1}(a \neq 0)$ or $\tilde{A}_{1}^{*}(a=0)$. Let $X$ be the double cover of $V$ branched along the union of $F_{1}$ and another irreducible fibre $F_{2} . X$ is an elliptic surface with two reducible fibres of type $\tilde{E}_{7}$ and a reducible fibre of type $\tilde{A}_{3}$ (or $\tilde{D}_{4}$ ). If $F_{2}$ is singular (this happens when $\mu a^{2}=4 \lambda$ ) it has an additional fibre of type $\tilde{A}_{1}$. The elliptic fibration has also two sections. We claim that $X$ has another elliptic fibration with two reducible fibres of type $\tilde{E}_{8}$ and $\tilde{D}_{9}$. To see this we assume for simplicity that $F$ is of type $\tilde{A}_{3}$. Let

$$
D=2 R_{0}+R_{1}+2 R_{2}+3 R_{3}+4 R_{4}+3 R_{5}+2 R_{6}+R_{7}
$$




$$
D^{\prime}=2 R_{0}^{\prime}+R_{1}^{\prime}+2 R_{2}^{\prime}+3 R_{3}^{\prime}+4 R_{4}^{\prime}+3 R_{5}^{\prime}+2 R_{6}^{\prime}+R_{7}^{\prime}
$$

be the reducible fibres of type $\tilde{E}_{7}$, let $F=E_{0}+E_{1}+E_{2}+E_{3}$ be the other reducible fibre. Without loss of generality we may assume that the two sections $S_{1}$ and $S_{2}$ intersect the fibre $D$ at $R_{1}$ and $R_{7}$, respectively, and the fibre $D^{\prime}$ at $R_{1}^{\prime}$ and $R_{7}^{\prime}$, respectively. Also $S_{1}$ intersects $F$ at $E_{0}$ and $S_{2}$ intersects $F$ at $E_{2}$. Now consider the following disjoint curves with self-intersection 0 :

$$
\begin{gathered}
D_{1}=3 R_{0}+2 R_{2}+3 R_{3}+6 R_{4}+5 R_{5}+4 R_{6}+3 R_{7}+2 S_{2}+R_{7}^{\prime}, \\
D_{2}=R_{5}^{\prime}+R_{0}^{\prime}+2 R_{4}^{\prime}+2 R_{3}^{\prime}+2 R_{2}^{\prime}+2 R_{1}^{\prime}+2 S_{1}+2 E_{0}+E_{1}+E_{3} .
\end{gathered}
$$

By Hodge's Index Theorem, the divisors $D_{1}$ and $D_{2}$ are linearly equivalent. They span a pencil which defines an elliptic fibration with fibre $D_{1}$ of type $\tilde{E}_{8}$ and fibre $D_{2}$ of type $\tilde{D}_{9}$.

Let $X(t ; a)$ be the elliptic surface obtained by the above construction when we take $F_{2}=F(\lambda, \mu)$ with $t=\lambda / \mu \neq 0, \infty$. The linear substitution $X \rightarrow c X, Y \rightarrow Y, Z \rightarrow c^{-1} Z$ extends to an isomorphism $X(t ; a) \cong X\left(c^{4} t, c^{2} a\right)$. Let the group $\mathbf{C}^{*}$ act on $\mathbf{C}^{*} \times \mathbf{C}^{*} \backslash\{(t, a)$ : $\left.a^{2}=t\right\}$ by the formula $(t, a) \rightarrow\left(c^{4} t, c^{2} a\right)$. The orbit space is isomorphic to $\mathbf{P}^{1} \backslash\{0,1, \infty\}$. When we vary $(t, a) \in \mathbf{C}^{*} \times \mathbf{C}$, we obtain a family $\mathcal{Y} \rightarrow \mathbf{C}^{*} \times \mathbf{C}$ of $M_{2}$-polarized K3 surfaces with $X(t ; a) \cong \mathcal{Y}_{(t, a)}$ for $a^{2} \neq 4 t, 0$. When $a^{2}=4 t, 0$, the fibre $\mathcal{Y}_{(t, a)}$ is singular but birationally isomorphic to $X(t ; a)$. The surface $X(t ; 0)$ has a fibre of type $\tilde{D}_{4}$ and its Picard number equals 20. The surface $X\left(a^{2} / 4, a\right), a \neq 0$, has a reducible fibre of type $\tilde{A}_{1}$ and its Picard lattice is isomorphic to $U \perp E_{8} \perp D_{9} \perp<-2>$. Let $f: \mathbf{C}^{*} \times \mathbf{C} \rightarrow \mathbf{C}$ given by the formula $(t, a) \rightarrow a^{2} / 4 t$. As in the previous example, we can descend the family $\mathcal{Y} \rightarrow \mathbf{C}^{*} \times \mathbf{C}$ to a family $\overline{\mathcal{Y}} \rightarrow \mathbf{C}$ of pseudo-ample $M_{2}$-polarized K3 surfaces with singular fibres over $0=f(t, 0)$ and $1=f\left(a^{2} / 4, a\right)$. The period map extends to an isomorphism $\mathbf{A}^{1} \rightarrow \mathbf{K}_{M_{2}}$ which sends 0 to the isomorphism class of the surface $X(0)$ and sends 4 to the isomorphism class of the surface $X(2)$. The latter surface is a pseudo-ample but not ample $M$-polarized K3 surface.

Example (7.10) $(n=3)$ We skip the details. We have

$$
M_{3} \cong U \perp E_{8} \perp E_{8} \perp<-6>.
$$

We consider a rational elliptic surface $V$ with a section, one reducible fibre $F_{1}$ of type $\tilde{E}_{6}$ and one reducible fibre $F_{2}$ of type $\tilde{A}_{2}$. To construct such a surface we take a plane nonsingular cubic $C$ and three inflection points on it lying on a line (this means that they add up to 0 in the group law on the cubic with an inflection point taken as the origin). Then we take the pencil of cubic curves spanned by $C$ and the union of the tangent lines at the three inflection points. After resolving the base points of this pencil we arrive at the surface $V$. The surface $X$ is obtained as a minimal nonsingular model of the double cover of $V$ branched over $F_{2}$ and a nonsingular fibre. The surface $X$ is an elliptic K3 surfaces with a section, two reducible fibres of type $\tilde{E}_{6}$ (Kodaira's $I V$ ) and one reducible fibre of type $\tilde{A}_{5}$ (Kodaira's $I_{5}$ ). Its Mordell-Weyl group is $\mathbf{Z} / 3$ and the sublattice of $\operatorname{Pic}(X)$ spanned by the section and components of the reducible fibres is isomorphic to $E_{6} \perp E_{6} \perp A_{5}$. By Lemma (7.4), we get that $\operatorname{Pic}(X)$ is a hyperbolic lattice of discriminant 6 . One can find 
another pencil on this surface with three reducible fibres of type $\tilde{E}_{8}, \tilde{E}_{7}$, and $\tilde{A}_{2}$. Since its discriminant equals 6 , it must coincide with $\operatorname{Pic} X$. On the other hand, it has the same discriminant group as the lattice $M_{3}$. By Nikulin's uniqueness results, we conclude that $\operatorname{Pic} X \cong M_{3}$.

Remark (7.11). The following remark may be appropriate. As we have already noticed in the previous section, the rational one-dimensional boundary components of $D_{M}$ correspond to rank 2 primitive isotropic sublattices $S$ of $N=M^{\perp}$. Each component contains in its closure the 0-dimensional boundary component defined by an isotropic vector $f \in S$. If $N=U \perp M_{n}$ and $f \in U$, then $S$ is determined by a primitive isotropic vector in $M_{n}$. Now if identify $M_{n}$ with $\operatorname{Pic}\left(X^{\prime}\right)$ for some ample $M_{n}$-polarized K3 surface $X^{\prime}$ from the mirror moduli space, we find a bijection between one-dimensional rational boundary components of $D_{M} / \Gamma_{M}$ containing the given 0-dimensional boundary component and isomorphism classes of elliptic fibrations on $X^{\prime}$. When $M=<2>$ or $<4>$ the list of $\Gamma_{M}$-orbits of two-dimensional isotropic sublattices of $N$ containing a given primitive isotropic vector is given in [37]. We find that $S_{N}^{\perp} / S$ can be isomorphic to one of the following lattices:

$$
\begin{gathered}
A_{1} \perp E_{8} \perp E_{8}, A_{1} \perp D_{16}, E_{7} \perp D_{10}, A_{17} \quad(M=<2>) . \\
E_{8}^{2} \perp<-4>, D_{16} \perp<-4>, E_{8} \perp D_{9}, E_{7}^{2} \perp A_{3}, D_{17}, D_{12} \perp D_{5}, \\
D_{8}^{2} \perp<-4>, A_{15} \perp A_{1}^{2}, E_{6} \perp A_{11} \quad(M=<4>) .
\end{gathered}
$$

In our interpretation we obtain that the mirror surfaces contain elliptic fibrations with reducible fibres of type:

$$
\begin{gathered}
\tilde{A}_{1}, \tilde{E}_{8}, \tilde{E}_{8} ; \quad \tilde{A}_{1}, \tilde{D}_{16} ; \quad \tilde{E}_{7}, \tilde{D}_{10} ; \quad \tilde{A}_{17} \quad(M=<2>) . \\
\tilde{E}_{8}, \tilde{E}_{8} ; \quad \tilde{D}_{16} ; \quad \tilde{E}_{8}, \tilde{D}_{9} ; \quad \tilde{E}_{7}, \tilde{E}_{7}, \tilde{A}_{3} ; \quad \tilde{D}_{17} ; \\
\tilde{D}_{12}, \tilde{D}_{5} ; \quad \tilde{D}_{8}, \tilde{D}_{8} ; \quad \tilde{A}_{15}, \tilde{A}_{1}, \tilde{A}_{1} ; \quad \tilde{E}_{6}, \tilde{A}_{11} \quad(M=<4>) .
\end{gathered}
$$

We have seen already a pencil of type $\tilde{E}_{8}+\tilde{E}_{8}+\tilde{A}_{1}$ on surfaces from the mirror family of $\mathbf{K}_{<2>}$ and the pencils of type $\tilde{A}_{1}+\tilde{A}_{1}+\tilde{A}_{15}$ and $\tilde{E}_{8}+\tilde{D}_{9}$ on surfaces from the mirror family of $\mathbf{K}_{<4>}$.

Similar computation is known for the case $M=<6>$ (see [39]). We have the following types of elliptic fibrations on surfaces from $\mathbf{K}_{M_{3}}$ :

$$
\begin{gathered}
\tilde{E}_{8}, \tilde{E}_{8} ; \quad \tilde{D}_{16} ; \quad \tilde{E}_{8}, \tilde{E}_{7}, \tilde{A}_{2} ; \quad \tilde{D}_{14}, \tilde{A}_{2}, \tilde{A}_{1} ; \quad \tilde{D}_{10}, \tilde{D}_{6} ; \quad \tilde{D}_{8}, \tilde{E}_{7}, \tilde{A}_{1} ; \\
\tilde{A}_{15}, \quad \tilde{E}_{6}, \tilde{E}_{6}, \tilde{A}_{5} ; \quad \tilde{A}_{11}, \tilde{D}_{5}, \tilde{A}_{1} ; \quad \tilde{A}_{9}, \tilde{D}_{7} .
\end{gathered}
$$

In example (7.10) we have seen an elliptic fibration of type $\tilde{E}_{6}, \tilde{E}_{6}, \tilde{A}_{5}$.

8. Toric hypersurfaces. Recall the following mirror construction of Batyrev [5] which generalizes the original construction of Green-Plesser. Let $\Delta \subset \mathbf{R}^{n}$ be a convex $n$-dimensional lattice polytope given by inequalities:

$$
\sum_{j=1}^{n} a_{i j} x_{j} \leq 1, i=1, \ldots, k,
$$


where $a_{i j} \in \mathbf{Z}$ (a reflexive polytope). Let $\Delta^{*}$ be the polytope equal to the convex hull of the vectors $l_{i}=\left(a_{i 1}, \ldots, a_{i n}\right)$. It is also a reflexive polytope. Let $\mathbf{P}_{\Delta}\left(\right.$ resp. $\left.\mathbf{P}_{\Delta^{*}}\right)$ be the corresponding toric variety, and $\mathcal{F}(\Delta)$ (resp. $\mathcal{F}\left(\Delta^{*}\right)$ ) be the family of hypersurfaces in $\mathbf{P}_{\Delta}$ (resp. $\mathbf{P}_{\Delta^{*}}$ ) defined by $\Delta$-nondegenerate (resp. $\Delta^{*}$-nondegenerate) Laurent polynomials. For $n \leq 4$ there exists a map $f: \tilde{\mathbf{P}}_{\Delta} \rightarrow \mathbf{P}_{\Delta}$ such that the proper transform of a general member of the family $\mathcal{F}(\Delta)$ is a Calabi-Yau manifold. A similar construction with $\Delta^{*}$ defines another family of Calabi-Yau manifolds. In the case $n=4$ the two families of Calabi-Yau 3-folds satisfy the first attribute of mirror symmetry: the dimension of the local moduli space for a member of the first family is equal to the Picard number of a member of the second family [5].

Consider the special case of Batyrev's construction when $\Delta$ is a 3 -dimensional simplex:

$$
\Delta(w)=\left\{\left(t_{0}, t_{1}, t_{2}, t_{3}\right) \in \mathbf{R}^{4}: \sum_{i=0}^{3} w_{i} t_{i}=0, t_{i} \geq-1, i=0, \ldots, 3\right\},
$$

where $w=\left(w_{0}, w_{1}, w_{2}, w_{3}\right)$ is a collection of four positive integers with greatest common divisor equal to 1 and such that $d=w_{0}+w_{1}+w_{2}+w_{3}$ is divisible by each $w_{i}$. Here we identify $\mathbf{R}^{3}$ with the hyperplane $\sum_{i=0}^{3} w_{i} t_{i}=0$. The toric space $\mathbf{P}_{\Delta}$ is the weighted projective space $\mathbf{P}(w)=\mathbf{P}\left(w_{0}, w_{1}, w_{2}, w_{3}\right)$. The family $\mathcal{F}(\Delta)$ is the family of quasi-smooth hypersurfaces of degree $d$ in $\mathbf{P}(w)$. One of its representatives is the surface

$$
x_{0}^{d_{0}}+x_{1}^{d_{1}}+x_{2}^{d_{2}}+x_{3}^{d_{3}}=0,
$$

where $d_{i}=d / w_{i}, i=0, \ldots, 3$. Let $\Pi$ be the finite abelian group of order $d_{0} d_{1} d_{2} d_{3} / d^{2}$ equal to the kernel of the homomorphism

$$
\left(\mu_{d_{0}} \times \mu_{d_{1}} \times \mu_{d_{2}} \times \mu_{d_{3}}\right) / \mu_{d} \rightarrow \mu_{d}, \quad g_{0}^{a_{0}} \cdots g_{3}^{a_{n}} \rightarrow g^{w_{0} a_{0}+\ldots+w_{3} a_{3}},
$$

where $\mu_{d_{i}}$ denotes the group of $d_{i}$-th roots of unity with generator $g_{i}$; the subgroup $\mu_{d}$ of the product is generated by $g=g_{0} g_{1} g_{2} g_{3}$. Then, by Corollary 5.5.6 of [5], the dual family $\mathcal{F}\left(\Delta^{*}\right)$ consists of quotients by $\Pi$ of the family of $\Pi$-invariant hypersurfaces of degree $d$ in $\mathbf{P}(w)$

$$
\sum_{w_{0} i_{0}+w_{1} i_{1}+w_{2} i_{2}+w_{3} i_{3}=d} a_{i_{0} i_{1} i_{2} i_{3}} x_{0}^{i_{0}} x_{1}^{i_{1}} x_{2}^{i_{2}} x_{3}^{i_{3}}=0 .
$$

Example (8.1). Let us consider the special case where $w=(1,1,1,1), d=4$. The family $\mathcal{F}(\Delta(w))$ is the family of quartic hypersurfaces in $\mathbf{P}^{3}$. Modulo projective transformation this family defines an open subset of the moduli space $\mathbf{K}_{<4>}$. The group $\Pi$ is isomorphic to $(\mathbf{Z} / 4)^{2}$ and its two generators act by the formula:

$$
\begin{aligned}
& g_{1}:\left(x_{0}, x_{1}, x_{2}, x_{3}\right) \rightarrow\left(x_{0}, \zeta x_{1}, x_{2}, \zeta^{3} x_{3}\right), \\
& g_{2}:\left(x_{0}, x_{1}, x_{2}, x_{3}\right) \rightarrow\left(\zeta x_{0}, x_{1}, \zeta^{3} x_{2}, x_{3}\right),
\end{aligned}
$$


where $\zeta$ is a primitive 4 -th root of unity. The $\Pi$-invariant family of quartics is the onedimensional family

$$
V(\lambda): x_{0}^{4}+x_{1}^{4}+x_{2}^{4}+x_{3}^{4}+4 \lambda x_{0} x_{1} x_{2} x_{3}=0
$$

The quotient $V(\lambda) / \Pi$ is isomorphic to the surface in $\mathbf{P}^{4}$ given by the equations:

$$
u_{0} u_{1} u_{2} u_{3}-u_{4}^{4}=0, \quad u_{0}+u_{1}+u_{2}+u_{3}+4 \lambda u_{4}=0 .
$$

If $\lambda^{4} \neq 1$, the surface $V(\lambda) / \Pi$ has six rational double points of type $A_{3}$. Let $V_{\lambda}$ be the family of $\mathrm{K} 3$ surfaces obtained by simultaneous resolution of singularities of the surfaces $V(\lambda) / \Pi, \lambda^{4} \neq 1$.

Theorem (8.2). The family of surfaces $V_{\lambda}$ is a family of $M_{2}$-polarized surfaces.

Proof. Consider the following four lines on the surface

$$
l_{i}: u_{i}=u_{3}=0, i=0,1,2, \quad l_{3}: u_{0}+u_{1}+u_{2}=u_{3}=0 .
$$

It is easy to check that the six points $P_{i j}=l_{i} \cap l_{j}, 0 \leq i<j \leq 3$, are the singular points of type $A_{3}$ of $V(\lambda) / \Pi$. Let $D_{i j}$ be the 6 exceptional divisors coming from a minimal resolution of singularities $V_{\lambda} \rightarrow V(\lambda) / \Pi$, and let $R_{i}, i=0, \ldots, 3$, be the proper inverse transforms of the lines. Each divisor $D_{i j}$ consists of three irreducible $(-2)$-curves with the intersection graph isomorphic to the Dynkin diagram of type $A_{3}$. Let $S$ be the sublattice of $\operatorname{Pic}(X)$ spanned by the curves $R_{i}$ and the irreducible components of the divisors $D_{i j}$. We shall show that $S \cong M_{2}$. Consider the divisor

$$
D=R_{0}+R_{1}+R_{2}+R_{3}+R_{4}+D_{01}+D_{12}+D_{23}+D_{03} .
$$

The linear system $|D|$ defines an elliptic fibration on $V_{\lambda}$ with reducible fibre $D$ of type $\tilde{A}_{15}$ (Kodaira's $I_{16}$ ). Let $E_{02}$ and $E_{13}$ be the irreducible components of the divisors $D_{02}$ and $D_{13}$ which are disjoint from the divisor $D$. They must be components of some reducible fibres of the elliptic fibration. Since the sublattice of $\operatorname{Pic}\left(V_{\lambda}\right)$ generated by irreducible components of fibres is of rank at most 19 , we have only two possibilities. Either $E_{02}, E_{13}$ are components of one fibre of type $\tilde{A}_{3}$, or there exist irreducible curves $E_{01}^{\prime}$ and $E_{12}^{\prime}$ such that $E_{02}+E_{02}^{\prime}$ and $E_{13}+E_{13}^{\prime}$ are two fibres of type $\tilde{A}_{1}$. In the first case we find that $\operatorname{Pic}\left(V_{\lambda}\right)$ is of rank 20. Since the family $(*)$ admits a degeneration $(\lambda=\infty)$ with infinite local monodromy, its image in the moduli space $\mathbf{K}_{S}$ is not a point. Thus for generic $\lambda$, $\operatorname{Pic}\left(V_{\lambda}\right)$ is of rank 19, and we have the second possibility. Let $E_{0}$ be the component of $D_{02}$ which intersects $R_{0}$. Then $E_{0}$ is a section of our fibration, and as such it must intersect the fibre $E_{13}+E_{13}^{\prime}$ at one point. Since it does not intersect $E_{13}$, it intersects $E_{13}^{\prime}$ with multiplicity 1 . Now we leave to the reader to verify that $V_{\lambda}$ admits another elliptic fibration which contains $E_{02}+E_{0}+E_{13}^{\prime}+D_{01}$ in its fibre of type $\tilde{D}_{9}$ and $D_{23}$ in its fibre of type $\tilde{E}_{8}$. Arguing as in Example (7.8), we deduce from this that $\operatorname{Pic}\left(X_{\lambda}\right)=S \cong M_{2}$.

Notice also that the period map

$$
p: \mathbf{A}^{1} \backslash\left\{\lambda: \lambda^{4}=1\right\} \rightarrow \mathbf{K}_{M_{2}}, \quad \lambda \rightarrow\left[X_{\lambda}\right]
$$


is of degree 4 . Indeed, the group $\mu_{4}$ of 4 th roots of unity acts on $\mathcal{F}$ by the formula $\lambda \rightarrow \rho \lambda$ so that $p$ factors through a map $p^{\prime}: \mathbf{A}^{1} \backslash\{1\} \rightarrow \mathbf{A}^{1}$. The map $p^{\prime}$ can also be extended to a map $\bar{p}^{\prime}: \mathbf{A}^{1} \rightarrow \mathbf{A}^{1}$ by sending 1 to the period of a minimal nonsingular model of the surface $V(1) / \Pi$. This surface represents the unique isomorphism class of pseudo-ample but not ample $M_{2}$-polarized K3 surface. One can show by computing the monodromy at infinity that $\bar{p}^{\prime}$ is an isomorphism.

Example (8.3). Let $w=(3,1,1,1), d=6$. The dual polyhedron $\Delta^{*}$ can be identified with the convex hull of the vectors $(1,0,0),(0,1,0),(0,0,1)$, and $(-1,-1,-3)$. The toric hypersurfaces defining the family $\mathcal{F}\left(\Delta^{*}\right)$ are given by the Laurent polynomials

$$
a T_{1}+b T_{2}+c T_{3}+d T_{1}^{-1} T_{2}^{-1} T_{3}^{-3}+e=0 .
$$

Multiplying both sides by $T_{1} T_{2} T_{3}^{3}$ and homogenizing, we obtain a projective model of $V \in \mathcal{F}\left(\Delta^{*}\right)$ defined by the equation

$$
a T_{1}^{2} T_{2} T_{3}^{3}+b T_{1} T_{2}^{2} T_{3}^{3}+c T_{1} T_{2} T_{3}^{4}+d T_{0}^{6}+e T_{0} T_{1} T_{2} T_{3}^{3}=0 .
$$

This model is not normal. To normalize it, we introduce a new variable $T_{4}=T_{0}^{2} / T_{3}$. Then a normal projective model can be given by the equations

$$
T_{1} T_{2}\left(a T_{1}+b T_{2}+c T_{3}+e T_{0}\right)+d T_{4}^{3}=0, \quad T_{0}^{2}=T_{4} T_{3}
$$

After some obvious linear transformation of the variables, we may assume that the generic member of $\mathcal{F}\left(\Delta(w)^{*}\right)$ is isomorphic to the surface $X_{\lambda}$ in $\mathbf{P}^{4}$ given by the equations

$$
u_{1} u_{2} u_{3}-u_{4}^{3}=0, \quad\left(\lambda u_{0}+u_{1}+u_{2}+u_{3}\right) u_{4}+u_{0}^{2}=0 .
$$

This is a double cover of the cubic surface $u_{1} u_{2} u_{3}-u_{4}^{3}=0$ in $\mathbf{P}^{3}$ branched along the union of two curves $C_{1}$ and $C_{2}$ cut out by the planes $u_{4}=0$ and $4\left(u_{1}+u_{2}+u_{3}\right)-\lambda^{2} u_{4}=0$, respectively. The cubic surface has three singular points which are cyclic singularities of type $A_{2}$. After we resolve them, and then resolve the base points of the pencil of elliptic curves spanned by the inverse transforms of the curves $C_{1}$ and $C_{2}$, we find a rational elliptic surface $V_{\lambda}$ with a singular fibre of type $\tilde{A}_{8}$ (originating from the curve $C_{1}$ ). Its double cover branched over this fibre and another fibre (originating from $C_{2}$ ) is birationally isomorphic to $X_{\lambda}$. After we resolve its singular points, we obtain an elliptic K3 surface $\bar{X}_{\lambda}$ with a reducible fibre of type $\tilde{A}_{17}$. The elliptic fibration has also three disjoint sections. They come from the three exceptional curves on $V_{\lambda}$ obtained from the resolution of the base points of the elliptic pencil on the nonsingular model of the cubic surface. Applying Lemma (7.7), we obtain that $\operatorname{Pic}\left(\bar{X}_{\lambda}\right)$ is a hyperbolic lattice of rank 19 and discriminant 2 . There is only one such lattice, up to isomorphism. This is the lattice $U \perp E_{8} \perp E_{8} \perp<-2>$. Thus the mirror family for $\mathbf{K}_{<2>}$ considered in Example (7.8) can be represented by the surfaces from the family $\mathcal{F}\left(\Delta(w)^{*}\right)$. Also observe that we have demonstrated the existence of two different elliptic fibrations on $X$ from the list given in Remark (7.11).

Example (8.4). In our next example we take

$$
w=(1,6,14,26), \quad d=42,\left(d_{1}, d_{2}, d_{3}, d_{4}\right)=(42,7,3,2) .
$$


In this case the group $\Pi$ is trivial, and according to Batyrev we should have the selfmirrored family. This is true for our mirrors too. The family is $\mathbf{K}_{M}$, where

$$
M \cong \check{M}=U \perp E_{8}
$$

We shall see the latter family again in the next example.

Example (8.5). Consider the affine surface

$$
x_{1}^{d_{1}}+x_{2}^{d_{2}}+x_{3}^{d_{3}}=0, \quad d_{1}^{-1}+d_{2}^{-1}+d_{3}^{-1}<1 .
$$

According to [24] the link space $L$ of the singular point 0 is diffeomorphic to the quotient $G /[\tilde{\Gamma}, \tilde{\Gamma}]$, where $G$ is the universal cover of $P S L(2, \mathbf{R})$ and $[\tilde{\Gamma}, \tilde{\Gamma}]$ is the commutator subgroup of the discrete group $\tilde{\Gamma}$ of $G$ isomorphic to an extension

$$
1 \rightarrow \mathbf{Z} \rightarrow \tilde{\Gamma} \rightarrow \Gamma\left(d_{1}, d_{2}, d_{3}\right) \rightarrow 1
$$

Here $\Gamma\left(d_{1}, d_{2}, d_{3}\right)$ is the Fuchsian subgroup of $P S L(2, \mathbf{R})$ of signature $\left(0 ; d_{1}, d_{2}, d_{3}\right)$. Let $K=\tilde{\Gamma} /[\tilde{\Gamma}, \tilde{\Gamma}]$. Its order is $d_{1} d_{2} d_{3} / d$, where $d=$ l.c.m. $\left(d_{1}, d_{2}, d_{3}\right)$. The quotient $L / K=$ $G / \tilde{\Gamma}\left(d_{1}, d_{2}, d_{3}\right)$ is the link space of a quasi-homogeneous triangle singularity $D_{d_{1}, d_{2}, d_{3}}$ (see $[\mathbf{1 0}, \mathbf{2 9}])$. There exist exactly 14 triples $d_{1}, d_{2}, d_{3}$ for which the singularity $D_{d_{1}, d_{2}, d_{3}}$ is isomorphic to the singularity at the origin of the affine surface $P(x, y, z)=0$, where $P$ is a quasi-homogeneous polynomial of degree $N$ with weights $\left(q_{1}, q_{2}, q_{3}\right)$ given in the following table:

$\begin{array}{lrrrrrr}\text { name } & \left(d_{1}, d_{2}, d_{3}\right) & \left(q_{1}, q_{2}, q_{3}\right) & \mathrm{N} & \left(d_{1}^{\prime}, d_{2}^{\prime}, d_{3}^{\prime}\right) & d_{0} & P(x, y, z) \\ Q_{10} & (2,3,9) & (6,8,9) & 24 & (3,3,4) & 18 & z^{2} x+y^{3}+x^{4} \\ Q_{11} & (2,4,7) & (4,6,7) & 18 & (3,3,5) & - & z^{2} x+y^{3}+y x^{3} \\ Q_{12} & (3,3,6) & (3,5,6) & 15 & (3,3,6) & 6 & z^{2} x+y^{3}+x^{5} \\ Z_{11} & (2,3,8) & (6,8,15) & 30 & (2,4,5) & 24 & y^{3} x+x^{5}+z^{2} \\ Z_{12} & (2,4,6) & (4,6,11) & 22 & (2,4,6) & 12 & y^{3} x+y x^{4}+z^{2} \\ Z_{13} & (3,3,5) & (3,5,9) & 18 & (2,4,7) & - & y^{3} x+x^{6}+z^{2} \\ S_{11} & (2,5,6) & (4,5,6) & 16 & (3,4,4) & - & z^{2} x+z y^{2}+x^{4} \\ S_{12} & (3,4,5) & (3,4,5) & 13 & (3,4,5) & - & y^{2} z+x z^{2}+x^{3} y \\ W_{12} & (2,5,5) & (4,5,10) & 20 & (2,5,5) & 10 & x^{5}+y^{4}+z^{2} \\ W_{13} & (3,4,4) & (3,4,8) & 16 & (2,5,6) & - & y^{4}+y x^{4}+z^{2} \\ K_{12} & (2,3,7) & (6,14,21) & 42 & (2,3,7) & 42 & x^{7}+y^{3}+z^{2} \\ K_{13} & (2,4,5) & (4,10,15) & 30 & (2,3,8) & 20 & y^{3}+y x^{5}+z^{2} \\ K_{14} & (3,3,4) & (3,8,12) & 24 & (2,3,9) & 12 & x^{8}+y^{3}+z^{2} \\ U_{12} & (4,4,4) & (3,4,4) & 12 & (4,4,4) & 4 & x^{4}+y^{3}+z^{3}\end{array}$

For each of the fourteen triples $\left(d_{1}, d_{2}, d_{3}\right)$ consider the family of hypersurfaces of degree $N$ in $\mathbf{P}\left(1, q_{1}, q_{2}, q_{3}\right)$ given by the equation

$$
Q(w, x, y, z)=P(x, y, z)+\sum a_{i j k} w^{N-i q_{1}-j q_{2}-k q_{3}} x^{i} y^{j} z^{k}=0
$$


where the monomials $x^{i_{1}} y^{i_{2}} z^{i_{3}}$ form a basis of the Jacobian algebra $\mathbf{C}[x, y, z] /$ (partials of $P$ ) of the polynomial $P$. There exists a morphism $Y \rightarrow \mathbf{P}\left(1, q_{1}, q_{2}, q_{3}\right)$ such that the proper inverse transforms of the quasi-smooth hypersurfaces $Q=0$ form a family $\mathcal{F}\left(d_{1}, d_{2}, d_{3}\right)$ of $M$-polarized K3 surfaces, where $M$ is the lattice of rank $d_{1}+d_{2}+d_{3}-2$ generated by vectors $e_{i}$ with $\left(e_{i}, e_{i}\right)=-2$ and $\left(e_{i}, e_{j}\right) \in\{0,1\}, i \neq j$, determined by the incidence graph $T_{d_{1}, d_{2}, d_{3}}$ of Dynkin type (for example $T_{2,3,5}$ corresponds to $E_{8}$ ). Note that the dimension of the family is equal to $\operatorname{dim} \mathbf{K}_{M}$. It is equal to the subscript in the first column minus 2 . The family $\mathcal{F}\left(d_{1}^{\prime}, d_{2}^{\prime}, d_{3}^{\prime}\right)$ corresponds to the mirror moduli space $\mathbf{K}_{\check{M}}$. The involution on the set of fourteen triples

$$
\left(d_{1}, d_{2}, d_{3}\right) \longleftrightarrow\left(d_{1}^{\prime}, d_{2}^{\prime}, d_{3}^{\prime}\right)
$$

is the so-called Arnold's Strange Duality (see [1,9]). If we take the triple $(2,3,7)$ corresponding to the singularity $K_{12}$ we obtain that $\mathcal{F}(2,3,7)$ coincides with the family $\mathcal{F}(\Delta(w))$ where $w=(1,6,14,21)$. It is self-dual with respect to Batyrev's duality and mirror duality.

On the other hand let us consider the 12-dimensional family $\mathcal{F}(3,3,4)$ corresponding to the singularity $K_{14}$. It coincides with the family $\mathcal{F}(\Delta(w))$, where $w=(1,3,8,12)$. The group $\Pi$ is of order 2 . The Batyrev dual is the 6-dimensional family $\mathcal{F}\left(\Delta(w)^{*}\right)$ obtained by dividing $\Pi$-invariant members of $\mathcal{F}(\Delta(w))$ by $\Pi$. The mirror family of $\mathcal{F}(\Delta(w))$ is the 8-dimensional family $\mathcal{F}(2,3,9)$ corresponding to the singularity $Q_{10}$.

Suppose $\left(d_{1}, d_{2}, d_{3}\right)$ is such that there exists an integer $d_{0}$ such that

$$
\frac{1}{d_{0}}+\frac{1}{d_{1}}+\frac{1}{d_{2}}+\frac{1}{d_{3}}=1
$$

This happens for 9 triples from the above list. Then we can consider the family $\mathcal{F}(\Delta(w))$ where $w_{i}=d / d_{i}, d=$ g.c.d. $\left(d_{0}, d_{1}, d_{2}, d_{3}\right)$. The group $\Pi$ is isomorphic to the group $K$ from above. The $\Pi$-quotients of surfaces from $\mathcal{F}(\Delta(w))$ are smoothings of the singularity $D_{d_{1}, d_{2}, d_{3}}$ and hence belong to the family $\mathcal{F}\left(d_{1}, d_{2}, d_{3}\right)$. This shows that the Batyrev dual family $\mathcal{F}\left(\Delta(w)^{*}\right)$ is a subfamily of $\mathcal{F}\left(d_{1}, d_{2}, d_{3}\right)$.

For example, $\mathcal{F}\left(\Delta(1,3,8,12)^{*}\right)$ is a subfamily of $\mathcal{F}(2,3,8)$ of dimension 9 corresponding to the singularity $Z_{11}$. Also, $\mathcal{F}\left(\Delta(1,4,5,10)^{*}\right)$ is a subfamily of $\mathcal{F}(2,4,5)$ of dimension 11 corresponding to the singularity $K_{13}$.

The exact relationship between the two mirror constructions seems to be the following. Let $X$ be a member of the family $\mathcal{F}(\Delta)$. Then $\operatorname{Pic}(X)$ contains the primitive sublattice generated by the image of the restriction homomorphism $\operatorname{Pic}\left(\tilde{\mathbf{P}}_{\Delta}\right) \rightarrow \operatorname{Pic}(X)$. Let $M_{\Delta}$ be the abstract lattice isomorphic to this lattice. One can show that $M_{\Delta} \cong \operatorname{Pic}(X)$ for general member $\mathcal{F}(\Delta)$ if and only if $\Delta$ satisfies the following condition: for any 1-dimensional face $\Gamma$ of $\Delta$,

$$
l^{*}(\Gamma)=l^{*}\left(\Gamma^{*}\right)=0,
$$

where $\Gamma^{*}$ is the dual one-dimensional face of $\Delta^{*}$, and $l^{*}(F)$ denotes the number of integral points in the interior of a face $F$ (see [2]).

Conjecture (8.6). The lattice $M_{\Delta}$ always contains a 1-admissible isotropic vector such that there exists a primitive embedding

$$
M_{\Delta^{*}} \subset \check{M}_{\Delta} .
$$


Moreover, the equality takes place if and only if condition $\left(^{*}\right)$ is satisfied.

This conjecture is confirmed by a result of Batyrev (unpublished) and Kobayashi [19] implying that

$$
\operatorname{rank} M_{\Delta}+\operatorname{rank} M_{\Delta^{*}} \leq 20 .
$$

Also, Kobayashi shows that $\operatorname{rank} M_{\Delta^{*}}=\operatorname{rank} \check{M}_{\Delta}$ if $(*)$ is satisfied. Finally, the conjecture is consistent with the examples from above. If $\Delta=\Delta(1,3,8,12)$, we have $M_{\Delta} \cong T_{3,3,4}$. Since $\mathcal{F}\left(\Delta^{*}\right)$ is a subfamily of $\mathcal{F}(2,3,8)$ we have $M_{\Delta^{*}} \cong T_{2,3,8}$. On the other hand $\check{M}_{\Delta}=T_{2,3,9}$ and obviously $T_{2,3,8}$ embeds naturally in $T_{2,3,9}$. In the second example where $\Delta=\Delta(1,4,5,10)$ we have $M_{\Delta^{*}} \cong T_{2,4,5}$ and $\check{M}=T_{2,5,5}$.

In some cases when $\left(^{*}\right)$ is not satisfied, it is still possible to find a polyhedron $\Delta^{\prime}$ satisfying $\left(^{*}\right)$ and such that $\mathcal{F}\left(\Delta^{\prime}\right)$ is a subfamily of $\mathcal{F}(\Delta)$. For example, it is always possible in the case of the fourteen families from Example (8.5) [19]. In this case, one can verify that $M_{\Delta^{\prime *}}=\check{M}_{\Delta^{\prime}}$.

Remark (8.7). There is a list of weighted projective K3-hypersurfaces with Gorenstein singularities first derived by Miles Reid (unpublished) and later rediscovered by Yonemura [42]). It consists of 95 families. It contains the family of quartic hypersurfaces and its mirror family represented by surfaces of degree 36 in $\mathbf{P}(7,8,9,12)$ (number 52 in the list of Yonemura).

9. Other examples. Here we consider the examples related to Enriques and Kummer surfaces.

Example (9.1). Let $F$ be an Enriques surface, and $p: X \rightarrow F$ be its K3-cover (see $[4,7])$. We have

$$
H^{2}(F, \mathbf{Z}) / \text { Tors } \cong \operatorname{Pic}(F) / \text { Tors } \cong E_{8} \perp U .
$$

Thus $p^{*}(\operatorname{Pic}(F))$ is a sublattice of $\operatorname{Pic}(X)$ isomorphic to $M=E_{8}(2) \perp U(2)$ and $X$ acquires a canonical structure of an $M$-polarized K3 surface. Since $M$ does not contain vectors $\delta$ with $(\delta, \delta)=-2$, we can choose $C(M)^{+}$to be equal to $V(M)^{+}$. Replacing $j: M \rightarrow \operatorname{Pic}(X)$ by $-j$, if needed, we may assume that $j\left(V(M)^{+}\right)$contains the class of an ample divisor $p^{*}(D)$, where $D$ is an ample divisor on $F$. Thus any marked Enriques surface $\left(F, \phi: H^{2}(F, \mathbf{Z}) /\right.$ Tors $\left.\rightarrow E_{8} \perp U\right)$ defines an ample $M$-polarized K3 surface $(X, j)$. Conversely, given such $(X, j)$, it defines an involution $\sigma$ on $H^{2}(X, \mathbf{Z})$ by setting $\sigma(v)=x$, for any $v \in j(M)$, and $\sigma(v)=-v$, for any $v \in(j(M))^{\perp}$. One can show that any two primitive embeddings of the lattice $M$ on $L$ differ by an isometry of $L$ (see [28]). Thus, we can choose a marking of $\phi: H^{2}(X, \mathbf{Z}) \rightarrow L$ such that $j_{\phi}=j$. Since the involution $\sigma$ leaves the period $H^{2,0}(X)$ of $X$ unchanged, by the Global Torelli Theorem (Corollary (3.2)), there is a unique involution $\tau$ of $X$ such that $\sigma=\tau^{*}$. By using the the Lefschetz fixed-point formula, it is not difficult to show that the set of fixed points of $\tau$ is empty (see [28], p.221). Thus $X=F /(\tau)$ is an Enriques surface together with a marking defined by descending the isomorphism $j: E_{8}(2) \perp U(2) \rightarrow \operatorname{Pic}(F)$ to the isomorphism $E_{8} \perp U \rightarrow \operatorname{Pic}(X)=\operatorname{Pic}(F)^{\tau}$. This esablishes a bijective correspondence between the isomorphism classes of marked Enriques surfaces and isomorphism classes of 
ample $M$-polarized K3 surfaces. In particular, $\mathbf{K}_{E_{8}(2) \perp U(2)}^{a}$ can be viewed as the moduli space of marked Enriques surfaces.

We may embed $E_{8}(2) \perp U(2)$ diagonally into $E_{8} \perp E_{8} \perp U \perp U$ to obtain that

$$
\left(E_{8}(2) \perp U(2)\right)_{L}^{\perp} \cong E_{8}(2) \perp U(2) \perp U .
$$

In particular, if we define the mirror lattice by taking $f \in U$, we obtain

$$
\check{M} \cong M .
$$

Thus the moduli space $\mathbf{K}_{M}$ is its own mirror. If we take $f$ from $U(2)$ instead, we obtain

$$
\check{M}=U \perp E_{8}(2) .
$$

One can show that the moduli space $\mathbf{K}_{U \perp E_{8}(2)}$ can be represented by the family of double covers of the plane branched along the union of two cubics.

Example (9.2). The mirror family for the family of nonsingular minimal models of the Kummer surfaces $X=\operatorname{Kum}(\mathrm{A})$ associated to principally polarized abelian surfaces $A$ is the family $\mathbf{K}_{M}$, where $M^{\perp}=U(2) \perp U(2) \perp<-4>$. This must be well known but let me give a proof due to J. Keum. By Theorem 1.4.14 from [31] the embedding $<2>\hookrightarrow U \perp U \perp U$ is unique. Therefore we may assume that the class $h$ of the polarization of $A$ is mapped to $e+f$ where $e, f \in U,(e, e)=(f, f)=0,(e, f)=1$. Therefore $T_{A}=\operatorname{Pic}(A)_{H^{2}(A, \mathbf{Z})}^{\perp} \cong U \perp U \perp<-2>$. On the other hand it follows from ([4], Chapter VIII, §5) that $T_{X}=\operatorname{Pic}(X)_{H^{2}(X, \mathbf{Z})}^{\perp} \cong T_{A}(2) \cong U(2) \perp U(2) \perp<-4>$.

Now if we take $f$ from a copy of $U(2)$ we get

$$
\check{M}=U(2) \perp<-4>\cong\left(\begin{array}{lll}
0 & 2 & 2 \\
2 & 0 & 2 \\
2 & 2 & 0
\end{array}\right) .
$$

The miror family is the moduli space of hypersurfaces of degree $(2,2,2)$ in $\mathbf{P}^{1} \times \mathbf{P}^{1} \times \mathbf{P}^{1}$. This family is equal to the family $\mathcal{F}(\Delta)$ where $\Delta=[-1,1]^{3} \subset \mathbf{R}^{3}$. As was shown by Batyrev the dual family $\mathcal{F}\left(\Delta^{*}\right)$ is the family $\mathbf{K}_{M^{\prime}}$ where $M^{\prime \perp}=U \perp \check{M}$. This family of K3 surfaces was studied by C. Peters and J. Stienstra in [33]. If we take $f \in U$, the dual moduli space of $\mathbf{K}_{M^{\prime}}$ is equal to $\mathbf{K}_{\check{M}}$. So the Kummer family and the Peters-Stienstra family share the same mirror family.

\section{References}

[1] V. Arnold, Critical points of smooth functions, Proc. I.C.M, Vancouver, 1974, pp. 18-39.

[2] P. Aspinwall, D. Morrison, String theory on K3 surfaces, 1994, IASSNS-hep-94/23

[3] P. Aspinwall, D. Morrison, Mirror symmetry and the moduli space of K3 surfaces, (to appear). 
[4] W. Barth, C. Peters, A. Van de Ven, Compact Complex Surfaces, Ergenbnisse der Mathematik und ihrer Grenzgebiete, 3. Folge, Band 4, Springer-Verlag, 1984.

[5] V. Batyrev, Dual polyhedra and mirror symmetry for Calabi-Yau hypersurfaces in toric varieties, J. Alg. Geometry, 3 (1994), 493-535.

[6] C. Borcea, K3 surfaces with involution and mirror pairs of Calabi-Yau manifolds, Rider College, preprint

[7] F. Cossec, I. Dolgachev, Enriques surfaces I, Birkhäuser. 1989.

[8] I. Dolgachev, V. Nikulin, Exceptional singularities of V. I. Arnold and K3 surfaces, Proc. USSR Topological Conference in Minsk, 1977.

[9] I. Dolgachev, Integral quadratic forms:applications to algebraic geometry, Sem. Bourbaki, 1982/83, $n^{\circ} 611$, Asterisque, vol. 105/106, Soc. Math. France, pp. 251-275.

[10] I. Dolgachev, On algebraic properties of algebras of automorphic forms, in "Modular Functions in Analysis and Number Theory", Lect. Notes in Mathematics and Statistics, vol. 5, Univ. Pittsburgh, 1983, pp. 21-29.

[11] Essays on Mirror Symmetry (ed. S.-T. Yau), Int. Press Co., Hong Kong, 1992.

[12] R. Fricke, Lehrbuch der Algebra, B. 3, Braunschweig, 1928.

[13] Géometrie des surfaces K3: modules et périodes, Astérisque, vol. 126, Soc. Math. France, 1985.

[14] A. Giveon, D.-J. Smit, Symmetries of the moduli space of (2,2) superstring vacua, Nucl. Phys. B349 (1991), 168-206.

[15] Ph. Griffiths, Periods of integrals on algebraic manifolds, I,II, Amer. J. Math. 90 (1968), 568-626, 805-865.

[16] Ph. Griffiths, L. Tu, Infinitesimal variation of Hodge structure, In 'Topics in Transcendental Algebraic Geometry", Ann. Math. Studies, vol. 106 , Princeton University Press, 1984.

[17] D. James, On Witt's Theorem for unimodular quadratic forms, Pac. J. Math. 26:2 (1968), 303-316.

[18] P.G. Kluit, On the normalizer of $\Gamma_{0}(N)$, in "Modular Functions of One Variable, V", Lect. Notes in Math., vol. 601, Springer, 1977, pp. 239-246.

[19] M. Kobayashi, Duality of weights, mirror symmetry and Arnold's strange duality, 1994, preprint

[20] J. Lehner, W. Newman, Weierstrass points of $\Gamma_{0}(n)^{*}$, Ann. Math., 79 (1964), 360-368.

[21] B. Lian, S.-T. Yau, Arithmetic properties of mirror map and quantum coupling, preprint, 1994, hep-th.

[22] B. Lian, S.-T. Yau, Mirror maps, modular relations and hypergeometric series II, preprint, 1994, hep-th.

[23] E. Martinec, Criticality, catastrophes, and compactifications, in "Physics and Mathematics of Strings", World Scientific, 1990,pp.389-433.

[24] J. Milnor, On the 3-dimensional Brieskorn manifolds, in "Knots, groups and 3manifolds", Ann. Math. Studies, vol. 84, Princeton Univ. Press 1975, pp. 175-224. 
[25] D. Morrison, On K3 surfaces with large Picard number, Invent. Math. 75 (1984), $105-121$.

[26] D. Morrison, Mirror symmetry and rational curves on quintic 3-folds: A guide for mathematicians, J. Amer. Math. Soc. 6 (1993), 223-247.

[27] M. Nagura, K. Sugiyama, Mirror symmetry of the K3 surface, Intern. J. Modern Physics A, vol. 10, No 2 (1995), 233-252.

[28] Y. Namikawa, Periods of Enriques surfaces, Math. Ann., 270 (1985), 201-222.

[29] W. Neumann, Abelian covers of quasihomogeneous singularities, in "Singularities", Proc. Symp. Pure Math., vol. 40, Part 2, A.M.S. Providence, 1983, pp. 233-243.

[30] V. Nikulin, Finite groups of automorphisms of Kähler K3 surfaces, Proc. Moscow Math. Society, 38(1980), 71-135.

[31] V. Nikulin, Integral quadratic forms and some of its geometric applications, Izv. Akad. Nauk SSSR, Ser. Math. 43 (1979), 103-167.

[32] V. Nikulin, On rational maps between K3 surfaces,in "Constantin Caratheodory: an international tribute", Vol. I, II, World Sci. Publishing. 1991, pp. 964-995.

[33] C. Peters, J. Stienstra, A pencil of K3 surfaces related to Apéry's recurrence for $\zeta(3)$ and Fermi surfaces for potential zero, in "Arithmetics of Complex Manifolds", Lect. Notes in Math., vol.1399, Springer-Verlag, 1989.

[34] H. Pinkham, Singularités exceptionnelles, la dualité étrange d'Arnold et les surfaces K-3, C.R. Acad. Sci. Paris, Ser. A-B, 284 (1977), 615-618.

[35] S.-S. Roan, Mirror symmetry and Arnold's duality, 1993, preprint MPI.

[36] Y. Ruan, G. Tian, A mathematical theory of quantum cohomology, Math. Res. Lett. 1, no. 2 (1994), 269-278.1994.

[37] F. Scattone, On the compactification of moduli spaces for algebraic K3 surfaces, Mem. A.M.S. 70 (1987). No. 374.

[38] G. Shimura, Introduction to the arithmetic theory of automorphic functions, Publ. Math. Soc. Japan, v. 11, 1971.

[39] H. Sterk, Lattices and K3 surfaces of degree 6, Lin. Alg. and Appl., 226-228 (1995), 297-309.

[40] A. Todorov, Some ideas from mirror geometry applied to the moduli space of K3, preprint.

[41] C. Voisin, Miroirs et involutions sur les surfaces K3, in "Journées de Géométrie Algébrique d'Orsay", vol. 218, Astérisque, Soc. Math. France, 1993, pp. 273-323.

[42] T. Yonemura, Hypersurface simple K3 singularities, Tôhoku Math. J. 42(1990), 351380 . 